\title{
Nopp140-chaperoned 2'-O-methylation of small nuclear RNAs in Cajal bodies ensures splicing fidelity
}

\author{
Jonathan Bizarro, ${ }^{1}$ Svetlana Deryusheva, ${ }^{2}$ Ludivine Wacheul, ${ }^{3}$ Varun Gupta, ${ }^{1}$ Felix G.M. Ernst, ${ }^{3}$ \\ Denis L.J. Lafontaine, ${ }^{3}$ Joseph G. Gall, ${ }^{2}$ and U. Thomas Meier ${ }^{1}$ \\ ${ }^{1}$ Albert Einstein College of Medicine, Bronx, New York 10461, USA $;{ }^{2}$ Carnegie Institution for Science, Baltimore, Maryland 21218 , \\ USA; ${ }^{3}$ RNA Molecular Biology, Fonds National de la Recherche Scientifique (FRS/FNRS), Université Libre de Bruxelles (ULB), \\ B-6041 Gosselies, Belgium
}

\begin{abstract}
Spliceosomal small nuclear RNAs (snRNAs) are modified by small Cajal body (CB)-specific ribonucleoproteins (scaRNPs) to ensure snRNP biogenesis and pre-mRNA splicing. However, the function and subcellular site of snRNA modification are largely unknown. We show that CB localization of the protein Nopp140 is essential for concentration of scaRNPs in that nuclear condensate; and that phosphorylation by casein kinase 2 (CK2) at $~ 80$ serines targets Nopp140 to CBs. Transiting through CBs, snRNAs are apparently modified by scaRNPs. Indeed, Nopp140 knockdown-mediated release of scaRNPs from CBs severely compromises 2'-O-methylation of spliceosomal snRNAs, identifying CBs as the site of scaRNP catalysis. Additionally, alternative splicing patterns change indicating that these modifications in U1, U2, U5, and U12 snRNAs safeguard splicing fidelity. Given the importance of CK2 in this pathway, compromised splicing could underlie the mode of action of small molecule CK2 inhibitors currently considered for therapy in cholangiocarcinoma, hematological malignancies, and COVID-19.
\end{abstract}

[Keywords: Cajal bodies; RNA modification; casein kinase 2; nuclear organization; phosphorylation; pre-mRNA splicing; scaRNPs; snRNA; snoRNPs]

Supplemental material is available for this article.

Received May 11, 2021; revised version accepted June 29, 2021.

Spliceosomal small nuclear RNAs (snRNAs) are the work horses at the core of the nuclear splicing machinery multiplying and diversifying the protein coding potential of the genome (Wilkinson et al. 2019). In addition to ribosomal RNAs (rRNAs) and transfer RNAs (tRNAs), snRNAs constitute the most abundant noncoding RNAs. These abundant snRNAs of the major spliceosome are U1, U2, U4, U5, and U6. All are modified at specific ribose moieties by 2'-O-methyl groups and at specific bases by isomerization of uridines to pseudouridines (Morais et al. 2021). Although it is known that pseudouridines in snRNAs are essential for proper assembly into snRNPs and for pre-mRNA splicing (Yu et al. 1998; Zhao and Yu 2004), the role of the 2'-O-methyl groups remains poorly defined. It has been particularly challenging to access the roles of snRNA modifications in intact cells. Here we uncover an explanation for their cellular functions.

The enzymes responsible for snRNA modifications have been identified: the small Cajal body (CB)-specific RNPs (scaRNPs). Two major classes of scaRNPs are distinguished by their H/ACA and C/D scaRNAs, which

Corresponding author: tom.meier@einsteinmed.org Article published online ahead of print. Article and publication date are online at http://www.genesdev.org/cgi/doi/10.1101/gad.348660.121. guide snRNA pseudouridylation and 2'-O-methylation, respectively, by site-specific base pairing (Maxwell and Fournier 1995; Smith and Steitz 1997; Kiss 2001). Each scaRNP consists of one of many distinct scaRNAs, of four core proteins, and of the CB-specifying protein WDR79 (also known as TCAB1). In case of H/ACA scaRNPs, the four core proteins include the pseudouridine synthase NAP57 (also known as dyskerin) and in case of C/D scaRNPs, the methyl transferase fibrillarin (Kiss 2004; Meier 2005). As the name suggests, scaRNPs are concentrated in CBs (Massenet et al. 2017; Meier 2017). Similarly, snRNAs accumulate in and transit through CBs. Based on this confluence of the scaRNP enzymes and their target snRNAs in CBs, CBs have long been implicated as the sites of snRNA modification (Darzacq et al. 2002). Indeed, exogenous snRNA constructs are only modified when targeted to CBs but not to nucleoli (Jády et al. 2003).

C 2021 Bizarro et al. This article is distributed exclusively by Cold Spring Harbor Laboratory Press for the first six months after the full-issue publication date (see http://genesdev.cshlp.org/site/misc/terms.xhtml). After six months, it is available under a Creative Commons License (Attribution-NonCommercial 4.0 International), as described at http://creativecommons.org/licenses/by-nc/4.0/. 
CBs were discovered $>100$ yr ago and have been known by various names, accessory body of the nucleolus, coiled bodies, and now CBs, but their function is still shrouded in mystery (Gall 2003; Machyna et al. 2013). Like many nuclear bodies, CBs are mainly defined by their composition. For example, they are enriched in coilin, a CB-specific protein of still unknown function, in snRNPs and scaRNPs, and in Nopp140, which also concentrates in nucleoli (Meier and Blobel 1994; Meier 2005). In coilin knockout cells, residual CBs remain that harbor Nopp140 and scaRNPs but not snRNPs supporting a scaRNP-specific function for Nopp140 in CBs (Tucker et al. 2001). More recently, formation of CBs and nucleoli have been assigned to the mechanism of liquid-liquid phase separation (Brangwynne et al. 2011; Zhu and Brangwynne 2015; Lafontaine et al. 2020). Indeed, the enrichment in CBs of RNAs, RNA binding proteins, and proteins with intrinsically disordered domains (e.g., Nopp140 and coilin) provide fertile ground for such a mechanism (Meier and Blobel 1994; Tantos et al. 2013; Neugebauer 2017; Na et al. 2018).

Nucleoli, the sites of rRNA synthesis and ribosome assembly, are by far the most phase-dense bodies of every cell. Their formation is apparently aided by liquid-liquid phase separation (Brangwynne et al. 2011; Zhu and Brangwynne 2015; Lafontaine et al. 2020). By transmission electron microscopy, three distinct regions are discernable, fibrillar centers (FCs) and the surrounding dense fibrillar component (DFC), which are altogether embedded in the granular component (GC). The rRNA genes are located in the FCs and transcribed at the border to the DFC into which the nascent rRNAs extend while being modified and processed (Hadjiolov 1985; Derenzini et al. 1990; Spector 1993; Scheer and Hock 1999; Dundr and Misteli 2001). Many subsequent steps of maturation and assembly with ribosomal proteins occur in the GC, where various forms of preribosomes are found before export to the cytoplasm. The home of small nucleolar RNPs (snoRNPs), which function in ribosomal RNA modification, are the most densely packed parts of nucleoli, the DFCs. Multiple factors are required in DFCs to orchestrate the dance between snoRNPs and nascent rRNAs while avoiding RNA tangles and electrostatic repulsion. Nopp140 and helicases are only some of such chaperones.

Nopp140 is a nucleolar (DFC) and Cajal body phosphoprotein encoded by the gene NOLC1 (Meier and Blobel 1990, 1994). Except for its association with snoRNPs and scaRNPs, its function is poorly defined. We now show that the phosphorylation of Nopp140 is required for its accumulation in CBs. High-resolution CRISPR screens identified Nopp140 as essential for cell survival (Hart et al. 2015; Wang et al. 2015). Using a targeted CRISPR/Cas9 approach in polyploid HeLa cells, we established three cell lines with very low levels of Nopp140 ( 1\%-7\% residual protein level); i.e., Nopp140 knockdown (KD) cell lines (Bizarro et al. 2019). Surprisingly, Nopp140 KD cells do not exhibit any growth or gross phenotypes. Nevertheless, the KD cells reveal subtle but clear differences in Nopp140 chaperoned activities filtering nonessential from essential functions. We showed that one of these nonessential functions is corralling scaRNPs in Cajal bodies (Bizarro et al. 2019). In Nopp140 low-expressing cells, all scaRNPs are released from Cajal bodies but the overall levels and integrity of the RNPs remain unaffected. As one of the consequences, the telomerase scaRNP is no longer sheltered in CBs but has continuous access to telomeres extending them gradually (Bizarro et al. 2019). Here we present the consequences of Nopp140 KD for all other scaRNPs when no longer maintained in CBs and for snoRNPs in nucleoli.

\section{Results \\ Establishment of stable Nopp140 rescue cells}

In a prior study, we generated three stable Nopp140 knockdown (KD) cell lines, KD1a, KD1b, and KD2 originating from two HeLa parent lines P1 and P2 (Bizarro et al. 2019). In the Nopp140 KD cells, intact scaRNPs were displaced from CBs. This phenotype could be rescued by transient re-expression of Nopp140 establishing that it was not an off-target effect of our CRISPR/Cas9 approach (Bizarro et al. 2019). To allow for biochemical and genome-wide approaches of Nopp140 rescue, we reintroduced Nopp140 on a plasmid with a selectable marker into the Nopp140 KD2 cells followed by antibiotic resistance selection of single clones to obtain three stable rescue cell lines, Nopp140 R2a, R2b, and R2c. Indirect immunofluorescence localized Nopp140 and NAP57, the pseudouridine synthase of H/ACA RNPs, in nucleoli and CBs (Fig. 1A, arrows) in the P2 parent cells (Fig. 1A, top). In contrast, in the Nopp140 KD2 knockdown cells, Nopp140 was lost from CBs and nucleoli whereas NAP57 was present in nucleoli but lost from CBs (Fig. 1A, middle). Nopp140 R2a rescue cells uniformly expressed Nopp140 in both nucleoli and CBs (arrows) and rescued the CB localization of NAP57 (Fig. 1A, bottom). According to fluorescent signal in nucleoli and CBs, all three rescue cell lines re-expressed Nopp140 to $60 \%-$ $80 \%$ of the parent cells (Fig. 1B). Surprisingly, when protein levels of Nopp140 in the rescue cells were assessed by Western blotting, Nopp140 re-expression appeared more subtle (Fig. 1C). Apparently, the different dynamic range of the two immunodetection methods is responsible for this discrepancy. This is supported by the fact that Nopp140 re-expression was increased $>13$-fold when assessed by Western blotting (Fig. 1D, cf. R2a-c and KD2) but only sevenfold when detected by indirect immunofluorescence (Fig. 1B).

Light microscopy did not detect any morphological differences between Nopp140 parent and KD cells, but alterations were noticed at the ultrastructural level (Bizarro et al. 2019). Differential contrast by electron microscopy identifies the classic tripartite structure of nucleoli, the light fibrillar centers (FCs) surrounded by the dense fibrillar component (DFC) that are altogether embedded in the granular component (GC) (Fig. 1E, P2, one FC-DFC unit is outlined). In the Nopp140 KD2 cells, the contrast of the DFC, where Nopp140 and snoRNPs reside, was lost (Fig. $1 \mathrm{E}, \mathrm{KD} 2$ ), but reappeared in the Nopp140 rescue cells 


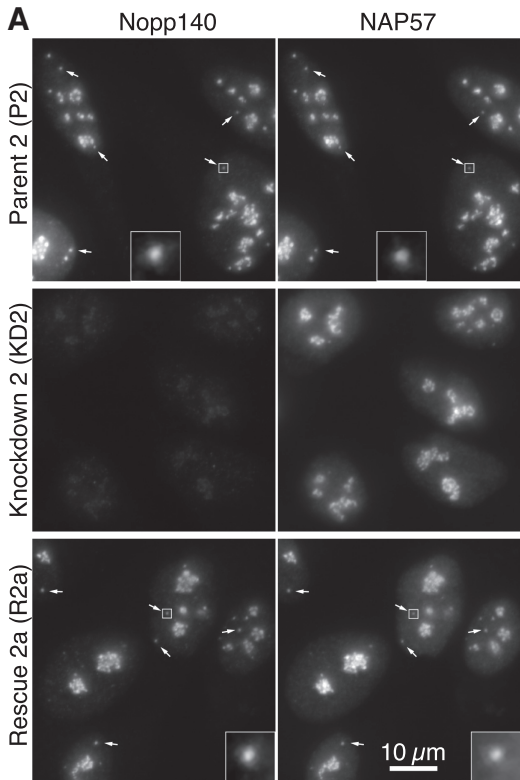

B Nopp140 signal in nucleoli and CBs

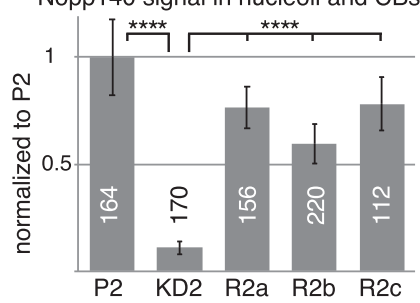

C

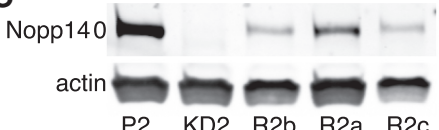

D Nopp140/actin western blot signal

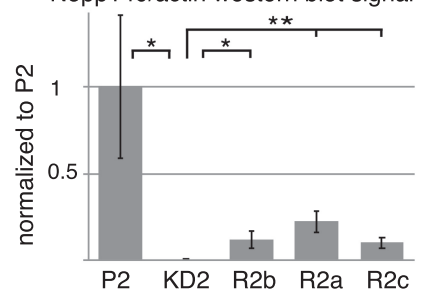

E Nucleoli
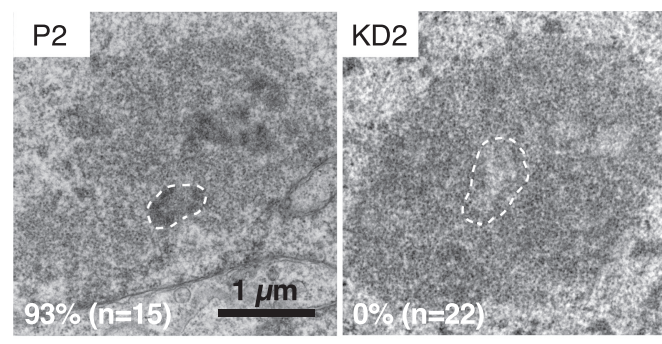

F CBs

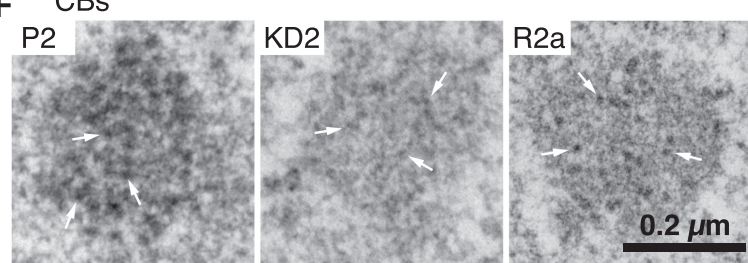

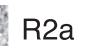

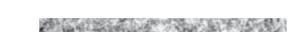

$\mathrm{R} 2 \mathrm{a}$

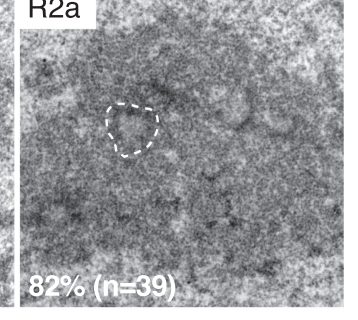

G Granule Size

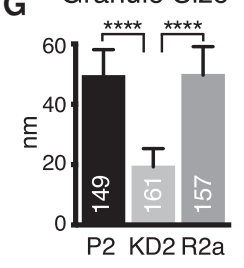

Figure 1. Effects of Nopp140 knockdown (KD) on nucleoli and Cajal bodies (CBs) are restored in cells stably re-expressing Nopp140. (A) Indirect immunofluorescence with Nopp140 (left) and NAP57 antibodies (right) on parent 2 (P2; top), KD2 (middle), and rescue 2a cells (R2a; bottom). Note that five of many CBs are highlighted in the top and bottom panels (arrows) and one CB is framed by a square and magnified fivefold (insets). Neither Nopp140 nor NAP57 is visible in CBs (middle panels) and Nopp140 is strongly reduced in nucleoli (left). Scale bar, $10 \mu \mathrm{m}$. (B) Quantification of Nopp140 fluorescent signal in nucleoli and $\mathrm{CBs}$ of $\mathrm{P} 2, \mathrm{KD} 2$, and rescue cells R2a-c normalized to P2 cells. The means \pm standard deviations (SDs) are indicated. The numbers refer to the number of cells analyzed for each cell line. $(* * * *) P<0.0001$. (C) Western blots of whole-cell extracts of the same set of cells probed for Nopp140 (top) and actin (bottom) and detected by a near-infrared imaging system (Odyssey). (D) Quantification of the near-infrared signals of triplicate Western blots shown in $C$ and normalized to P2 signals. Means \pm SDs are shown. $\left(^{*}\right) P<0.05,\left({ }^{* *}\right)$ $P<0.005$. (E) Transmission electron micrographs of $\mathrm{P} 2$ (left), KD2 (middle), and R2a (right) nucleoli. One fibrillar center (FC) surrounded by a dense fibrillar component (DFC) is outlined in each nucleolus (dashed line). Note the striking loss of contrast of the DFC in the KD2 and its reappearance in the R2a cells re-expressing Nopp140. The percentage of nucleoli with distinct DFCs for each cell line is indicated in the bottom left of each panel with the number analyzed in parentheses. Percentages vary significantly $(P<0.0001)$ between $\mathrm{P} 2$ and KD2 and between KD2 and R2a cells but not between P2 and R2a cells $(P>0.3)$. Scale bar, $1 \mu \mathrm{m} .(F)$ Transmission electron micrographs of one CB from each of the same cells as in $E$. Three electron-dense granules are pointed out in each CB (arrows). Scale bar, $0.2 \mu \mathrm{m}$. $(G)$ Quantification of CB granule size for each cell line. Mean \pm SD. $\left(^{* * * *}\right) P<0.0001$. The number of granules analyzed is indicated within each column. Note the significant reduction in granule size in the KD2 cells and their restoration in the rescue cells R2a (and R2c). There was no significant difference in granule number per CB between the cell lines $(P>0.3)$. In all cases, significance was assessed by unpaired $t$-tests.

(Fig. 1E, R2a). In case of CBs, the loss of scaRNPs caused a marked reduction in contrast and a halving in size of the granules making up their coils (Fig. 1F, cf. KD2 and $\mathrm{P} 2)$. In contrast, CB granule size and contrast in the rescue cells was mostly restored (Fig. 1F, R2a). Quantification of CB granule size confirmed our observations (Fig. 1G). Together with the repopulation of CBs with scaRNPs in the rescue cells, these data further indicate that scaRNPs normally reside in the granules of CBs. We previously reported the same effects of Nopp140 $\mathrm{KD}$ on nucleoli and CBs in other KD cells, KDla, demonstrating that this is not a clonal aberration (Bizarro et al. 2019). Throughout this study, we used three Nopp140 rescue cell lines to document that the observed effects are not due to some unrelated event but specifically to Nopp140 depletion.

\section{Phosphorylation of Nopp140 is required for $C B$ localization}

The most outstanding feature of the Nopp140 amino acid sequence is its 10 alternating acidic serine and positively charged lysine-, alanine-, and proline-rich repeats (Meier and Blobel 1992). Casein kinase 2 (CK2) is responsible for the phosphorylation of $\sim 80$ serines in the 10 acidic serine stretches effecting a $40-\mathrm{kDa}$ shift in migration on denaturing polyacrylamide gels (SDS-PAGE) (Meier 1996; Meier and Blobel 1992). It is this extreme phosphorylation of Nopp140 that forms the basis for its interaction with scaRNPs and snoRNPs because dephosphorylated Nopp140 no longer associates with the RNPs (Wang et al. 2002). Given that scaRNPs are lost from CBs concomitantly with Nopp140, we tested whether 
phosphorylation of Nopp140 by CK2 is required for CB accumulation. We first tested whether simple inhibition of CK2 affected localization of Nopp140. After evaluating 5,6-dichloro-1- $\beta$-D-ribofuranosylbenzimidazole (DRB) and quinalizarin, which caused morphological changes in the nucleolus and cell death (Scheer et al. 1984; Cozza et al. 2009), we used the small molecule CX-4945 (silmitasertib), a selective ATP-competitive inhibitor of CK2 (Siddiqui-Jain et al. 2010). Even after $3 \mathrm{~d}$ of incubation with the inhibitor, when most cells had died, Nopp140 and NAP57 localization was unaffected in the surviving cells where they remained in CBs and nucleoli (Fig. 2A, bottom panels). To ascertain that Nopp140 lost its phosphorylation during the incubation period, migration of Nopp140 on SDS-PAGE was analyzed by Western blotting (Fig. 2B). Surprisingly, at all time points of CK2 inhibition, Nopp140 migrated at $140 \mathrm{kDa}$ and not at the $100 \mathrm{kDa}$ characteristic for the unphosphorylated protein (Fig. 2B, ar- row). This indicated that the phosphorylation of Nopp140 did not turn over while the CK2 inhibitor caused cell cycle arrest (Siddiqui-Jain et al. 2010). Hence, we tested whether the CK2 inhibitor prevented only phosphorylation of newly synthesized Nopp140. Taking advantage of our Nopp140 KD cells, which express little to no Nopp140 (Fig. 2C, lane 1), Nopp140 was transiently transfected in the absence and presence of the CK2 inhibitor CX-4945. The phosphorylation status of Nopp140 was assessed by its migration on SDS-PAGE. Thus, newly synthesized and fully phosphorylated Nopp140 was only detected in the absence of CK2 inhibitor (Fig. 2C, lane 2). However, the inhibitor mostly prevented Nopp140 phosphorylation, which migrated at its unphosphorylated position of $100 \mathrm{kD}$ (Fig. 2C, lane 3). Additionally, some more slowly migrating bands, representing intermediate degrees of phosphorylation, were observed. Therefore, while leaving already phosphorylated Nopp140 untouched, the CK2
A
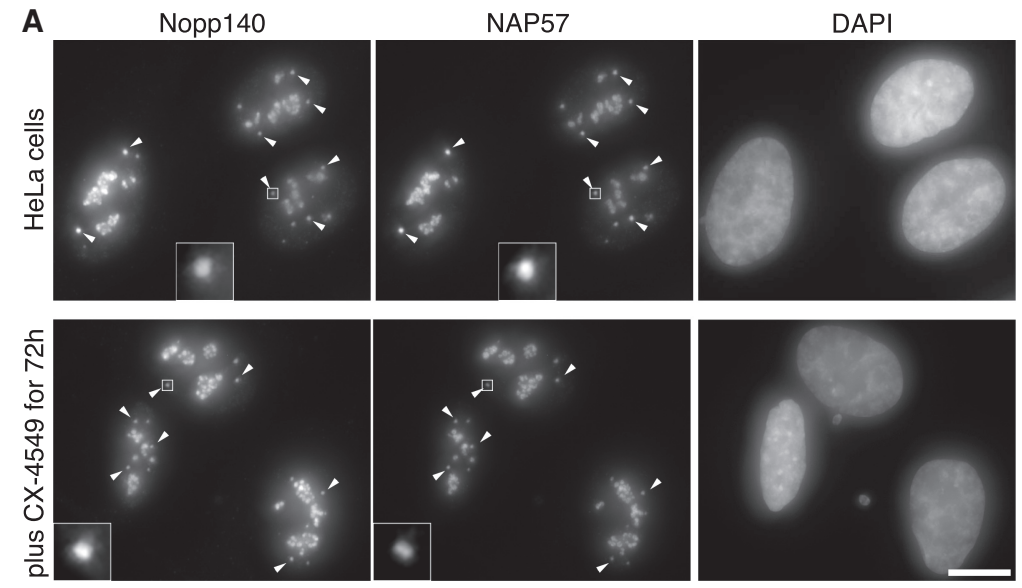

B

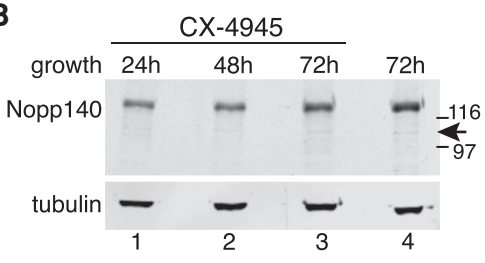

C

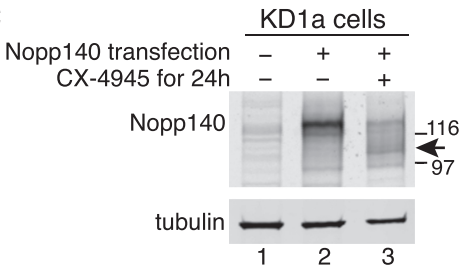

D
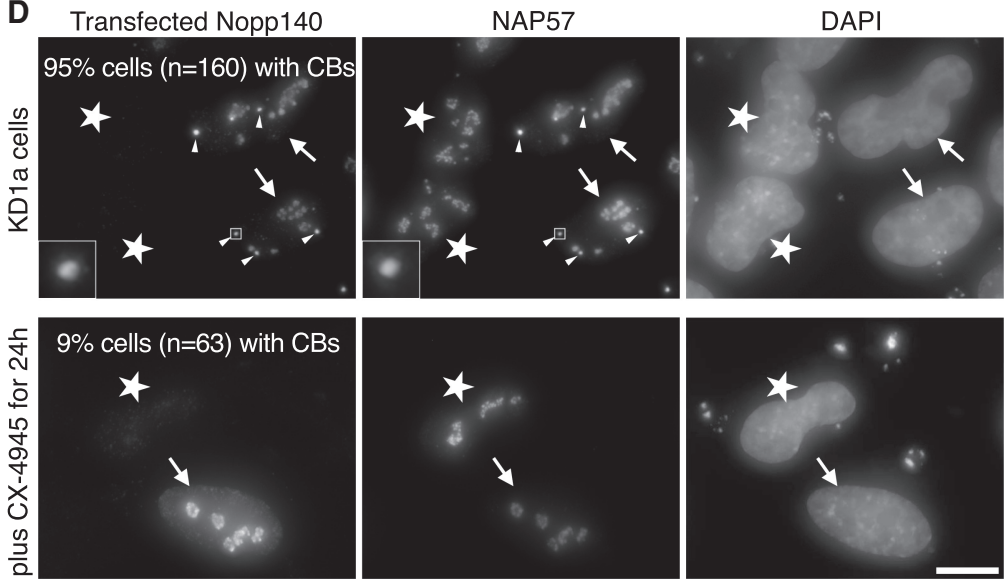

Figure 2. Nopp140 phosphorylation by casein kinase $2(\mathrm{CK} 2)$ is required for CB localization. $(A, t o p$ panels) Indirect immunofluorescence for Nopp140 (left) and NAP57 (middle) with DAPI DNA stain (right) on control P2 cells. (Bottom panels) The same, but after a $72 \mathrm{~h}$ incubation with $10 \mu \mathrm{M}$ concentration of the CK2 inhibitor CX-4945 (silmitasertib). Note that both proteins remain in nucleoli and CBs under both conditions. Some CBs are highlighted (arrowheads) and one $\mathrm{CB}$ is framed by a square and magnified fivefold (insets). Scale bar, 10um. $(B$, top $)$ Western blots on whole P2 cell lysates as described for Figure 1C after incubation for various numbers of hours with $10 \mu \mathrm{M}$ CX-4945 (lanes 1-3) and without (lane 4). The migrating positions of Nopp140 and tubulin are indicated (left), that of unphosphorylated Nopp140 (right, arrow), and that of molecular weight markers (116 and $97 \mathrm{kDa})$. (C) Western blots of KDla cell extracts after 24-h transient transfection with Nopp140 (lanes 2,3) or untransfected (lane 1), with (lane 3) and without (lanes 1,2) $10 \mu \mathrm{M}$ CX-4945. Labeling as in $B$. ( $D$, top panels) Indirect immunofluorescence of KD1a cells transfected (arrows) or untransfected (stars) with Nopp140 and stained for Nopp140 (left) and NAP57 (middle) with DNA stain (right). Note that transfected Nopp140 localizes to both nucleoli and CBs in these Nopp140 knockdown cells, whereas endogenous NAP57 localizes to nucleoli independent of the presence of Nopp140 but to CBs only in Nopp140 transfected cells (five CBs are highlighted by arrowheads). (Bottom panels) Same as top but in the presence of the CK2 inhibitor CX4945. Note that newly synthesized and unphosphorylated Nopp140 accumulates only in nucleoli but not CBs and, accordingly, NAP57 is not recruited to CBs either. The percentage of Nopp140 transfected cells with obvious Nopp140- and NAP57-positive CBs is indicated in the top of the left panels. Percentages vary significantly between cells untreated (top) or treated (bottom) with the CK2 inhibitor CX-4945 $(P<0.0001$, unpaired $t$-test $)$. Where visible, one $\mathrm{CB}$ is framed by a square and magnified fivefold (insets). Scale bar, $10 \mu \mathrm{M}$. 
inhibitor effectively prevented phosphorylation of newly synthesized Nopp140. To investigate the localization of Nopp140 and NAP57 under these conditions, we performed indirect immunofluorescence experiments (Fig. 2D). In the absence of the CK2 inhibitor, transfection of Nopp 140 (cells with arrows) caused both proteins to localize to CBs and nucleoli. However, in the residual untransfected Nopp140 KD cells (asterisks), NAP57 localized only to nucleoli but not CBs (Fig. 2D, top panels) consistent with our previous results (Bizarro et al. 2019). In the presence of CX-4945, newly translated, unphosphorylated Nopp140 similarly accumulated only in nucleoli but not CBs (Fig. 2D, bottom panels, arrow) demonstrating that Nopp140 phosphorylation was required for CB targeting. Consistent with the fact that NAP57 accumulation in CBs depends on the localization of Nopp140 in CBs (Bizarro et al. 2019), NAP57 stayed in nucleoli but was excluded from CBs in the presence of CX-4945, even in Nopp140 transfected cells (Fig. 2D, bottom panels, arrow). In summary, CK2 phosphorylation of Nopp140 is required for the accumulation of both proteins in CBs and by extension for that of scaRNPs.

Thus, the molecular consequences of displacement of scaRNPs from CBs, described in the remainder of this manuscript, need to be considered in CX-4945 (silmitasertib) therapy. Silmitasertib is currently in a phase II trial of cholangiocarcinoma (NCT02128282) as well as being considered as a drug against hematological malignancies and COVID-19 (Chon et al. 2015; Bouhaddou et al. 2020; SilvaPavez and Tapia 2020). As outlined below, compromised splicing fidelity due to reduced 2'-O-methylation of snRNAs may thus contribute to the molecular mode of action of this CK2 inhibitor.

\section{ScaRNP depletion from CBs alters snRNA modification}

Due to the conspicuous colocalization in CBs of scaRNP enzymes and their snRNA targets, CBs have long been presumed the subcellular sites of snRNA modification, but this was never demonstrated for endogenous particles in mammalian cells (Darzacq et al. 2002; Jády et al. 2003; Deryusheva et al. 2012). After having documented the consequences of telomerase displacement from CBs, we investigated the consequence of displacement of all other scaRNPs from CBs. In Nopp140 KD cells, scaRNPs remained intact and their cellular levels unaltered indicating that the bulk of scaRNPs in CBs separated from their snRNP targets and dispersed in the nucleoplasm (Bizarro et al. 2019). We thus asked whether and to what extent snRNAs were still modified in Nopp140 KD cells.

We started with analysis of U2, which with 14 pseudouridines and ten 2'-O-methyl groups is the most highly modified spliceosomal snRNA (Morais et al. 2021). In fact, onequarter of its $5^{\prime}$-half nucleotides are modified. The modifications are important for snRNP biogenesis and premRNA splicing ( $\mathrm{Yu}$ et al. 1998; Dönmez et al. 2004; Zhao and Yu 2004). To map modified residues of snRNAs, we isolated total RNA from parent and Nopp140 KD cells and performed several reverse transcriptase-based assays after chemical treatment of the RNA with CMC [N-cyclo-
hexyl-N'-(2-mopholinoethyl) carbodiimide metho-p-toluene sulfonate] to identify pseudouridines and in the presence of low dNTP concentrations to recognize 2'-O-methyl groups (Bakin and Ofengand 1993; Maden et al. 1995). Under these conditions, strong stops are observed during RT revealing pseudouridines and 2'-O-methylated residues (Fig. 3A-E).

Using fluorescent primer extension on CMC-derivatized U2 snRNA, we first mapped pseudouridines (Fig. 3A). As the peak size of none of the 9 pseudouridines varied significantly between the two parent cells (Fig. 3A, blue) and the three knockdown cells (Fig. 3A, red tones), we conclude that pseudouridylation of U2 snRNA was unaffected by Nopp140 KD. Quantification of the first strong stop marking the pseudouridine at residue 60 of U2 snRNA (Fig. 3A, $\Psi 60$ ) confirmed the same degree of pseudouridylation of parent and KD cells (Fig. 3D). Using an independent assay, we globally mapped the pseudouridines of CMC-derivatized U2 snRNA by semiquantitative RT-PCR(Fig. 3E, top panel, green). Indeed, RT-PCR across $\Psi 89$ and $\Psi 91$ and a stretch including seven $\Psi$ s of U2 snRNA ( $\Psi 43-91$ ) confirmed the same RT termination rate between the parent and Nopp140 KD cells relative to that of a $3^{\prime}$ unmodified stretch (Fig. 3E, F3/F2 relative to F1). Quantification of the amplified bands normalized to U2 snRNA from the parent cells confirmed our conclusions (Fig. 3D). In summary, U2 snRNA is fully pseudouridylated, even in the absence of scaRNP pseudouridylases from CBs where U2 snRNA remains concentrated (Bizarro et al. 2019). Apparently, pseudouridylation of snRNAs is too important to be lost and instead occurs in the nucleoplasm of Nopp140 KD cells.

Next, we assessed the degree of ribose methylation of $\mathrm{U} 2$ snRNA. This was performed using low dNTP concentrations during reverse transcription with two different reverse transcription enzymes (RTs) that show differential sensitivity toward individual sites of 2'-O-methylation. In contrast to pseudouridines, the levels of all mapped 2'-O-methyl groups were significantly reduced in U2 snRNA isolated from $\mathrm{KD}$ cells (Fig. 3B,D, traces in red tones) relative to those from parent cells (Fig. 3B,D,blue traces). Quantification of the termination rate at Am30 (Fig. 3B) and Um47 (Fig. 3C) verifies the reduction of 2'O-methylation at those sites of U2 snRNA in Nopp140 $\mathrm{KD}$ versus parent cells (Fig. 3D). We further confirmed the results for U2-Am30 using an independent semiquantitative RT-PCR assay using EpiScript RT at low dNTP concentration, which is mainly sensitive to the $2^{\prime}$-Omethyl group at A30 but not those further downstream (Fig. 3E, bottom panels). The amplification efficiency across Am30 (Fig. 3E, bottom panels, F4+R) was compared with that of an unmodified stretch of U2 further downstream $(\mathrm{F} 1+\mathrm{R})$. Normalized quantification of the results for all parent and KD cells corroborated those obtained through fluorescent primer extension analysis (Fig. 3D). Remarkably, the levels of 2'-O-methylation at Am30 and Um47 mirrored the degree of Nopp140 KD in those cells (Bizarro et al. 2019); i.e., lowest levels were detected in U2 snRNA from KD1a and KD2 cells, whereas intermediate levels were detected in those from the KD1b cells (Fig. 

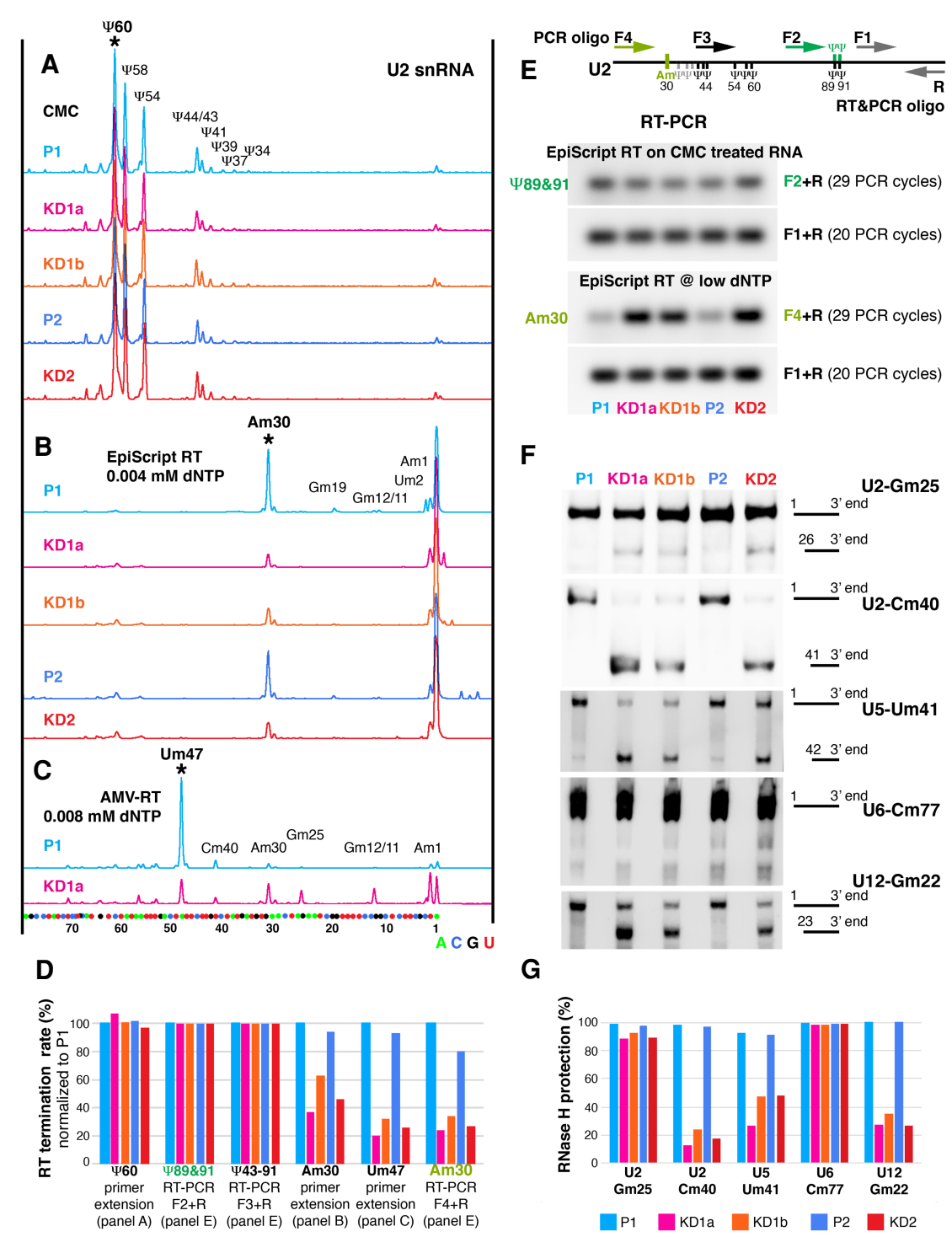

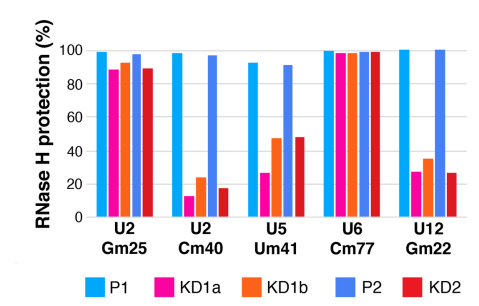

Figure 3. 2'-O-methylation, but not pseudouridylation, is reduced at most sites of RNA polymerase II transcribed snRNAs. $(A-C)$ Mapping of modified nucleotides in U2 snRNA of Nopp140 parent (P1 and P2; blue tones) and knockdown cells (KDla, $\mathrm{KD} 1 \mathrm{~b}$, and KD2; red tones) using fluorescent primer extension. (Bottom) Nucleotide positions were aligned to sequencing reactions on in vitro transcribed U2 snRNA. (A) Nine pseudouridines are detectable through strong stops after CMC treatment. Note the same height of all peaks in total RNAs isolated from all five cell lines, indicating undisturbed pseudouridylation of U2 snRNA after Nopp140 KD. (B) The 2'-O-methylated residue Am30 of U2 is readily detectable through a strong stop using EpiScript RT at low dNTP concentration in the parent traces (blue tones) but is severely reduced in the KD traces (red tones). Due to the initial strong stop at Am30, the subsequent 2'-O-methylated residues could not be determined reliably. $(C)$ The 2'-O-methylated residue Um47 is identified by a strong stop using AMV-RT at low dNTP levels in U2 from P1 (blue) and is severely reduced in KD1 cells (pink). Subsequent 2'-O-methylated residues are masked due to the strong initial stop. (D) Quantification of RT termination rates normalized to P1 (i.e., the degree of modification) of the indicated residues in $A-C$ (peaks marked by asterisks) and in $E$ (green and olive). Additionally, the termination rates across seven $\Psi$ s of U2 snRNA ( $\Psi 43-91)$ are quantified (primers F3 and $\mathrm{R} 1$ in schematic of $E$ ). Note the greater reduction in KDla (pink) over KDlb (orange) mirroring the residual Nopp140 levels and the lack of effect on any of the $\Psi$ s tested (key in G). (E) Semiquantitative RT-PCR of U2 using EpiScript RT on CMC derivatized RNA with primers $\mathrm{F} 2$ and $\mathrm{R}$ to detect $\Psi 89$ and $\Psi 91$ (green) and at low dNTP concentration with primers F4 and to detect 2'-O-methylated Am30 (olive). The amplification scheme is depicted at the top and the gels of the PCR products are below. Note that EpiScript RT does not detect 2'-O-methylated residues upstream of Am30 (shown in B). RNA from all cell lines was analyzed and the results expressed relative to the amplification products of an unmodified stretch of U2 (primers F1 and R) are quantified in $D .(F)$ RNase H protection assays to quantitatively detect the degree of 2'-O-methylation of snRNAs from all parent and KD cell lines at U2-Gm25, U2- Cm40, U5-Um41, U6-Cm77, and U12-Gm22. Note, the more full-length products are detected the more the specific site is 2'-O-methylated. $(G)$ Percent of RNase H protection for each cell line and nucleotide in $F$. Note, most experiments were performed two to three times and all numbers for $D$ and $G$ are given in Supplemental Table S1.

3D). Thus, the loss of 2'-O-methylation at U2-Am30 and Um47 correlated with the loss of Nopp140 and scaRNPs from CBs indicating that this snRNA modification normally occurs in CBs and is not fully required under standard conditions for growth in our cell lines.

To test whether the loss of 2'-O-methylation was specific to U2 snRNA, we used a quantitative site-specific assay on U5-Um41, U6-Cm77, and U12-Gm22, in addition to U2-Gm25 and U2-Cm40. Specifically, we used RNase H-mediated site-specific cleavage with chimeric RNA/ DNA oligos (Fig. 3F). In this assay, cleavage of RNA is prevented when the ribose at the specific residues is $2^{\prime}-\mathrm{O}$ - methylated (Yu et al. 1997). In the Nopp140 KD cells KD1a, KD1b, and KD2, methylation of U2-Cm40, U5Um41, and U12-Gm22 was mostly lost; i.e., cleavable (Fig. 3F). In contrast, in the parental cell lines P1 and P2, no observable cleavage was noted at those nucleotides indicating complete 2'-O-methylation (Fig. 3F). Remarkably, 2'-O-methylation at one residue of U2 (Gm25) and of U6 (Cm77) was unaffected in all cells (Fig. 3F). In the case of U6 snRNA this was not surprising because its 2'-O-methylation guide RNPs concentrate in nucleoli and not CBs (Ganot et al. 1999). The level of RNase H protection at these five sites of the four snRNAs was 
quantified (Fig. 3G). Importantly, the loss of 2'-O-methylation was due to that of Nopp140 because the modification of U2-Cm40, U2-Cm61, U5-Um41, and U12-Gm22 was fully restored in Nopp140-re-expressing cell lines (Supplemental Table S1). Additionally, the degree of loss of methylation again mirrored that of Nopp140 (Bizarro et al. 2019|; i.e., more loss from KD1a than KD1b snRNAs (Fig. 3G). Finally, methylation at G25 of U2 snRNA seems too vital to be lost and apparently occurs in the nucleoplasm of Nopp140 KD cells.

\section{RiboMethSeq captures most 2'-O-methylation sites in $s n R N A s$}

To corroborate and expand our findings of changes in 2 '-Omethylation of stable RNAs in Nopp140 KD cells, we used the systematic mapping approach of RiboMethSeq (RMS). This approach takes advantage of differential sensitivity to alkaline cleavage of 2 -O-methylated versus unmethylated residues that becomes statistically apparent during deep sequencing of alkaline cleaved abundant RNAs (Birkedal et al. 2015; Krogh et al. 2016; Marchand et al. 2016; Sharma et al. 2017). This method is particularly efficient in identifying the degree of 2'-O-methylation in rRNA but, as described below, also allows monitoring these modifications in other abundant RNAs, such as snRNAs (Krogh et al. 2017).

In fact, with a sequencing depth of 25 million reads per sample, RMS was sufficiently sensitive to reliably identify most 2'-O-methylated residues in the major spliceosomal snRNAs U1, U2, U5, and U6 (Fig. 4; Supplemental Table S1). However, coverage of U4 was insufficient to provide statistically significant scores in our sequencing. The RMS score (fraction of a residue that is 2'-O-methyl- ated) of most residues of snRNAs from the parental P2 cell lines was equal to or above 0.7 (Fig. 4A, blue; Supplemental Table S1). In contrast, the RMS scores for most residues of U1, U2, and U5 snRNAs from the Nopp140 KD2 cells were $<0.7$ (Fig. 4A, red; Supplemental Table $\mathrm{S} 1)$ yielding ratios of KD2 over $\mathrm{P} 2$ of $<0.6$ indicating a significant reduction in modification of those residues. In contrast, the $\mathrm{KD} 2 / \mathrm{P} 2$ ratios of all five $2^{\prime}$-O-methylated residues of U6 snRNA were $>0.7$, indicating that $2^{\prime}$-O-methylation of U6 was not or barely affected by Nopp140 KD. This confirmed our RNase $\mathrm{H}$ cleavage-based results for U6-Cm77 (Fig. 3F,G) and supports U6 modification occurring outside CBs (Ganot et al. 1999; Deryusheva and Gall 2019). Interestingly, in contrast to five other U2 residues, 2'-O-methylation of U2-G12 and U2-G25 was barely impacted by Nopp140 KD (Fig. 4A,B). For U2-Gm25, this lack of effect on 2'-O-methylation was also noted by RNase H-mediated cleavage with RNA/DNA hybrid oligonucleotides in all three Nopp140 KD cell lines (Fig. 3F,G; Supplemental Table S1). The robustness of these results was confirmed by an independent second round of RMS (Fig. 4B, P2'/KD2'). Importantly, the loss of methylation at all snRNA sites was restored in our rescue cells establishing it as a consequence of Nopp140 KD (Fig. 4B, R2a'). All individually determined and RMS scores of snRNA modification agree with each other and are numerically summarized in Supplemental Table S1. Apparently, the 2'-O-methylation of U2-Gm12 has to be added to that of U2-Gm25 as too vital to be lost or being mediated by a yet to be identified chaperone (Fig. 4A-C; Supplemental Table S1). We conclude that the loss of scaRNPs from CBs impacts 2'-O-methylation, but not pseudouridylation, of snRNAs transiting through these condensates.
A RMS scores of $\mathrm{P} 2$ and $K D 2$ snRNAs

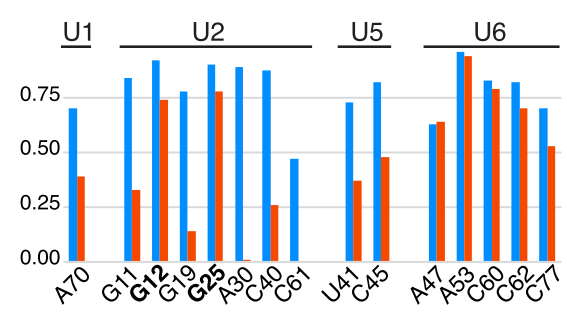

C

18S rRNA 2'-O-methylation
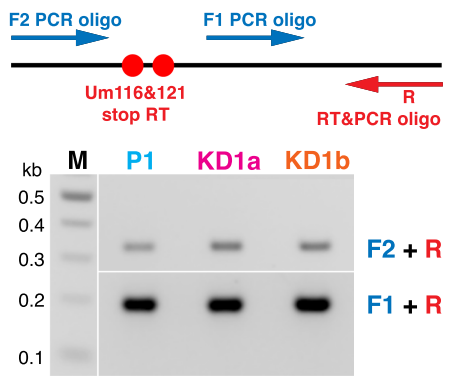

B

P2 P2' KD2 KD2' R2a'

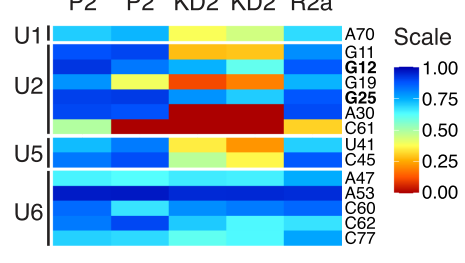

D $\quad$ 18S rRNA Um116\&121

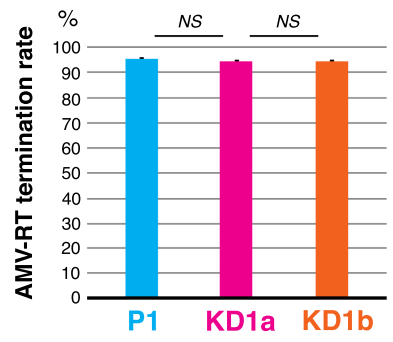

Figure 4. RiboMethSeq (RMS) shows a severe reduction of 2'-O-methylation at most sites of $\mathrm{U} 1, \mathrm{U} 2$, and $\mathrm{U} 5$ snRNAs but not $\mathrm{U} 6(A-C)$, or at Um116 and Um121 of $18 \mathrm{~S}$ rRNA detected by RT-PCR $(D, E)$. (A) Histogram of RMS scores for U1, U2, U5, and U6 snRNAs obtained from RMS of total RNA isolated from Nopp140 parent (P2, blue) and knockdown (KD2, red) cells. Note the reduction in 2'-O-methylation at all sites except those in U6 and at $\mathrm{Gm} 12$ and $\mathrm{Gm} 25$ of U2 snRNA (bold). (B) Heat map of RMS scores of snRNAs from two experiments on total RNA isolated on separate occasions from P2, KD2, and rescue cells (R2a). The prime indicates a separate experiment. Note the remarkable agreement of the RMS scores from the two experiments (P2 vs. $\mathrm{P}^{\prime}$ and $\mathrm{KD} 2$ vs. $\left.\mathrm{KD} 2^{\prime}\right)$ and the rescue of the loss of 2'-O-methylation after re-expression of Nopp140 (R2a'). (C) Semiquantitative RT-PCR of $18 \mathrm{~S}$ rRNA at low dNTP concentration with primers F2 and R relative to unmodified rRNA with primers $\mathrm{F} 1$ and $\mathrm{R}$ to detect 2'-O-methylated Um116 and 121 on P1 and KD1a and KD1b cell RNA. Schematic is at the top and gel of products is at the bottom. $(D)$ Histogram of percent of AMV-RT termination rates of triplicate experiments expressed as the ratio of intensities of the two bands in $C$ corrected for fragment length $($ mean $\pm \mathrm{SD})$. 


\section{2'-O-methylation of rRNA remains mostly unaffected}

In addition to scaRNPs in CBs, Nopp140 also associates with snoRNPs in nucleoli. There, snoRNPs are responsible for the modification of rRNA. In the absence of any obvious impact on ribosome synthesis, one of the most remarkable hallmarks of the Nopp $140 \mathrm{KD}$ cells is the reorganization of nucleoli evidenced by loosening or loss of contrast of the nucleolar DFC (Fig. 1E, KD2; Bizarro et al. 2019). It is in this compartment where Nopp140 and all snoRNPs normally concentrate and modify nascent preribosomal RNA guided by site-directed base pairing. To assess the impact on rRNA 2'-O-methylation, we first used our semiquantitative RT-PCR approach described above for U2 snRNA (Fig. 3E). Specifically, methylation at residues U116 and U121 of 18S rRNA was investigated in our Nopp140 parental P1 and KD1a and b cell lines (Fig. 4C). No difference in amplification efficiency was noted between all three RNA sample templates and across the methylated region versus an unmodified stretch of 18S rRNA (Fig. 4C, bottom). Quantification of the AMV-RT termination rates confirmed that Nopp140 KD had no impact on 18S rRNA methylation at residues Um116 and Um121 (Fig. 4D). This result indicated that at the tested positions 2'-O-methylation of rRNA, unlike that of snRNA, remained unaffected by Nopp140 KD.

To systematically survey the impact of Nopp140 KD on pre-rRNA 2'-O-methylation at each position known to be modified, we next used RMS. Previous studies reported 2'-O-methyl modification scores of rRNA for different cancer cell lines under various conditions including transient KD of fibrillarin, the methyltransferase of C/D snoRNPs (Krogh et al. 2016; Erales et al. 2017; Incarnato et al. 2017; Sharma et al. 2017; Taoka et al. 2018). To assess the impact of Nopp140 KD on rRNA methylation, we considered in duplicates the ratio of RMS scores of the knockdown (KD2 and KD2') over the parental (P2 and P2') cell rRNAs. The ratios of most modified residues were $>0.8$ (Supplemental Table S2, framed red). To ascertain a true effect, we used an extra margin by considering a ratio of $\leq 0.7$ as reduced. Thus, out of 38 robustly modified residues of $18 \mathrm{~S}$ rRNA in all studies, only five residues (13\%) were affected by Nopp140 KD (Supplemental Table S2, 18S colored, framed red). All but one (Supplemental Table S2, $18 \mathrm{~S}$ orange) of those five residues were constitutively hypomodified (RMS score $\leq 0.7$ ) in at least one of the prior studies (Supplemental Table S2, 18S yellow; Krogh et al. 2016; Erales et al. 2017; Sharma et al. 2017; Taoka et al. 2018). Out of the 65 robustly modified residues of $28 \mathrm{~S}$ rRNA in all studies, 2'-O-methylation of 12 residues $(18 \%)$ was reduced in Nopp140 KD cells (Supplemental Table S2, 28S colored, framed red). All but two of those 12 were constitutively hypomodified or, in the case of Cm2422, the RMS score ratio was significantly reduced $(\leq 0.7)$ after fibrillarin KD in at least one of the prior studies (Supplemental Table S2, 28S yellow; Krogh et al. 2016; Erales et al. 2017; Sharma et al. 2017; Taoka et al. 2018). Only three of the residues identified as hypomodified in most studies were unaffected by Nopp140 KD (Supple- mental Table S2, 28S, green, Gm1316, Cm1881, and Um2415). Nevertheless, most of the rRNA modifications that were reduced after Nopp140 KD corresponded to hypomodified or naturally sensitive sites of methylation (Krogh et al. 2016; Erales et al. 2017; Sharma et al. 2017). Given the normal proliferation of those cells, this suggested that these sites may be less important for ribosome assembly and function. However, the most interesting changes in rRNA methylation are those three residues in $18 \mathrm{~S}$ and 28S rRNA that are normally fully modified in all studies, even under transient fibrillarin depletion; i.e., 18S-Um428, 28S-Gm4370, and 28S-Cm4456 (Supplemental Table S2, orange). Apparently, the chaperoning role of Nopp140 is particularly critical for those snoRNPs that are responsible for guiding methylation at those sites. A heat map representation of the RMS score tables for all rRNAs visually confirms the above points (Fig. 5A). The affected residues are marked with yellow and orange dots and the unaffected but hypomodified residues are indicated with green dots following the color scheme of Supplemental Table S2 (Fig. 5A). The heat map further underscores the reproducibility of the two parent (P2 and $\mathrm{P}^{\prime}$ ) and knockdown RMS scores (KD2 and KD2'). Importantly, Nopp140 re-expression restored the levels of 2'-O-methylation at the affected sites of rRNA to those of parent cells (Fig. 5A, R2 $\mathrm{a}^{\prime}$ ). Unsupervised clustering of the RMS scores groups together the knockdown, the parent, and the rescue cells (Fig. 5B). Most of the methylation sites affected by Nopp140 KD (Fig. 5B, red in the dendrogram) cluster with the hypomodified sites in rRNA (Fig. 5B).

To assess a possible impact on ribosome biogenesis of these subtle but reproducible changes in rRNA 2'-Omethylation, we investigated pre-rRNA processing by Northern blotting with probes for specific processing intermediates on total RNA from parent and KD cells (Fig. 5C). Specifically, we used probes for the internal transcribed spacer 1 (ITS1) and 2 (ITS2) and for the $5^{\prime}$-external transcribed spacer (5' ETS) (Fig. 5C). No significant variations in mature $28 \mathrm{~S}$ and $18 \mathrm{~S}$ rRNAs and in pre-rRNA processing were detected between the parent and KD cell lines (Fig. 5C). We further examined whether the changes in rRNA 2'-O-methylation impacted the localization of the rRNAs themselves or of snoRNAs. For this purpose, we grew parent (Nopp140-positive) and KD cells (Nopp140-negative) on the same dish in a 1:1 mixture (Fig. 5D). We detected 28S rRNA, ITS2, H/ACA snoRNAs (E3, ACA8, ACA18, and ACA28 combined), and the C/D snoRNA U3 by RNA FISH (Fig. 5D, both panels, green) together with immunofluorescence for Nopp140 (Fig. 5D, right panels, red) to identify parent (Fig. 5D, orange nucleoli) and KD cells (Fig. 5D, green nucleoli, KD). Note the localization of $28 \mathrm{~S}$ rRNA in both its place of synthesis (nucleoli) and of function (cytoplasmic ribosomes) (Fig. 5D). In contrast, ITS2 was restricted to nucleoli, its place of synthesis and processing. As expected, neither 28S nor ITS2 rRNAs were detected in CBs (Fig. 5D, arrowheads in Nopp140-positive cells). Localization of none of the RNAs changed between parent and KD cells (Fig. 5D). The absence of any difference in these assays was perhaps 
A

A P2 P2' KD2 KD2' R2a'

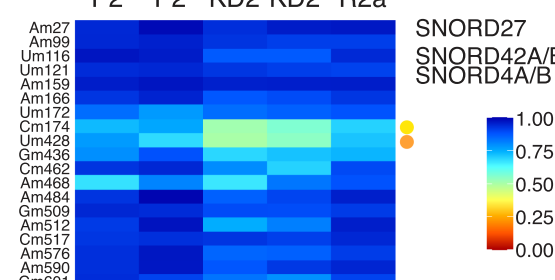

$\underline{18 S}$

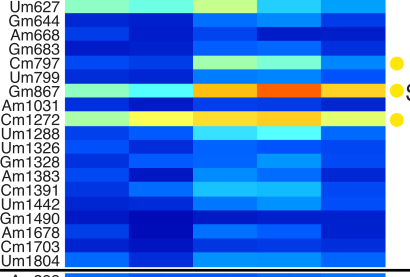

Am398
Am400
Gm1316

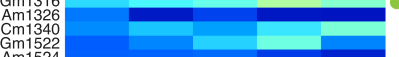

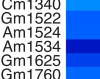

Am18
Cm18
$\mathrm{Cm} 183$
$\mathrm{Am} 23$
$\mathrm{C} 23 \mathrm{~m}$

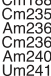

51
51
63
01
15
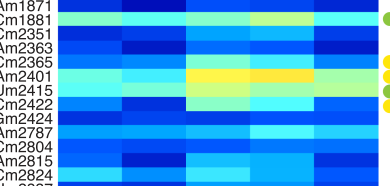

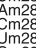

$\mathrm{Cm} 286$
$\mathrm{Gm} 28$
$\mathrm{C} m 370$
$\mathrm{Am} 37$

$\mathrm{Cm} 370$
$\mathrm{Am} 37$
$\mathrm{Am} 37$
$\mathrm{Gm} 374$

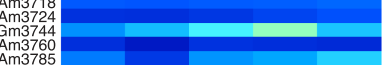

Am3
Gmin3
$\mathrm{Cm} 38$
$\mathrm{Um} 38$

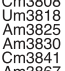

Ams81
Ams367
Cmm3869

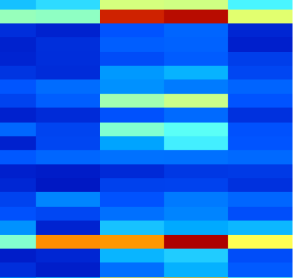

-

SNORD102

SNORD55

SNORD50A

$\underline{28 S}$

SNORD15A
SNORD10

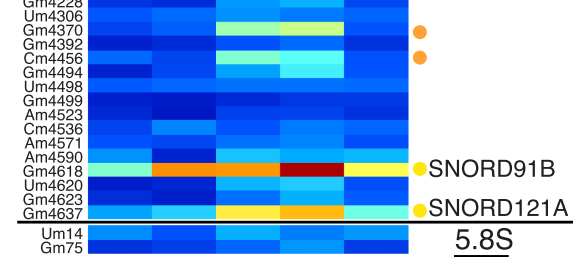

B

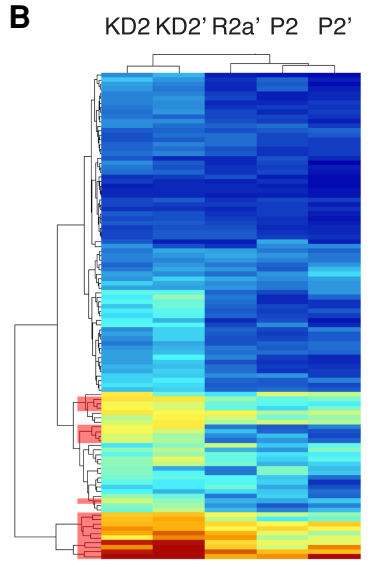

C

A: P1

B: KD1a

$\mathrm{C}: \mathrm{KD} 1 \mathrm{~b}$

$\mathrm{D}: \mathrm{P} 2$

$\mathrm{E}: \mathrm{KD} 2$

rRNA ITS1
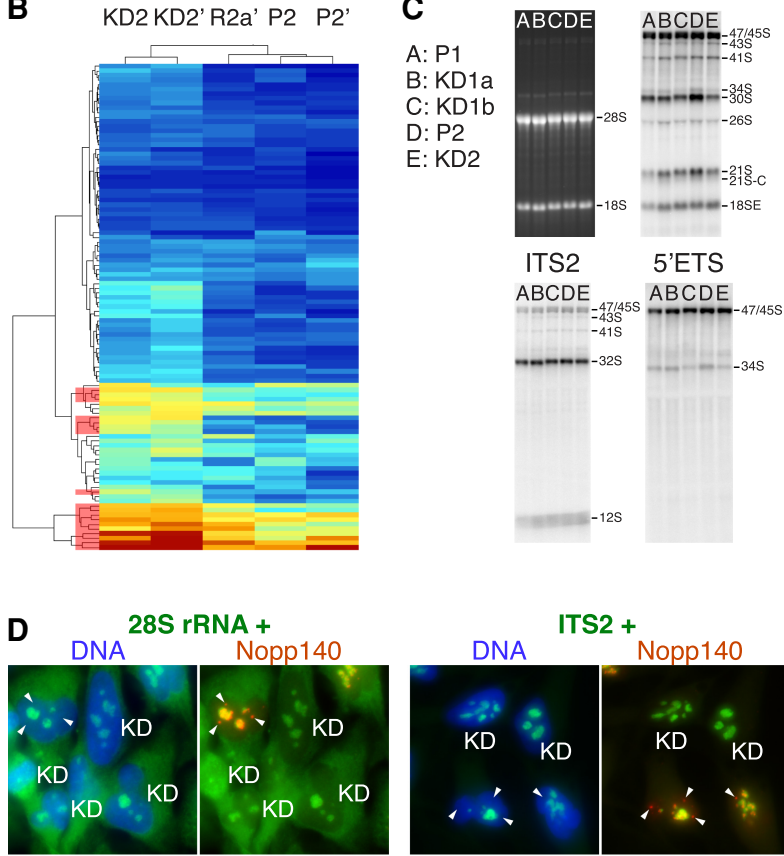

H/ACA snoRNAs + DNA

Nopp140
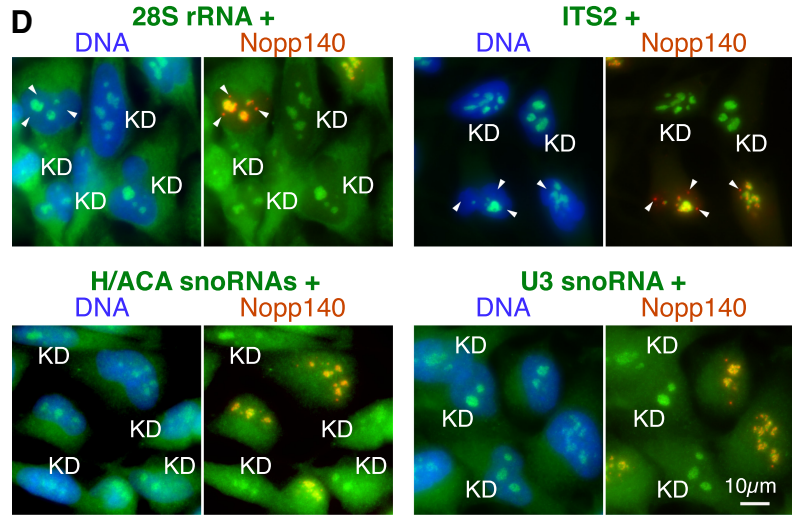

U3 snoRNA +

DNA Nopp140

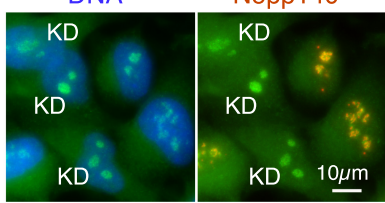

E
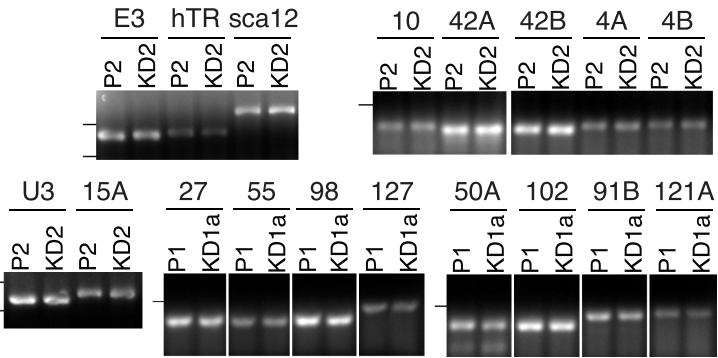

Figure 5. RiboMethSeq (RMS) of rRNAs shows reduced 2'-O-methylation at select sites without consequences for pre-rRNA processing localization, or steady-state levels of snoRNAs and mature rRNAs between parent and KD cells. (A) Heat map of RMS scores from two experiments as in Figure 4A but of rRNAs. Sites of reduced 2'-O-methylation are indicated with yellow or, if normally fully modified, with orange dots. Normally hypomethylated nucleotides that are unaffected by Nopp140 KD are indicated with green dots following the color scheme of Supplemental Table S2. The snoRNAs tested for their abundance in $E$ are indicated by name next to the nucleotide they are complementary to for site-directed modification. $(B)$ Unsupervised clustering of the values in $A$ groups the KD cells together and shows the sites of reduced 2'-O-methylation (red in the dendrogram at the left) to segregate with the normally hypomodified residues in the parent and rescue cells. (C) Mature rRNA and pre-rRNA processing analysis. Total RNA ( $3 \mu \mathrm{g})$ from the indicated cell lines was separated on denaturing agarose gels, stained with ethidium bromide, to detect mature $18 \mathrm{~S}$ and 28S rRNAs, or processed for Northern blotting with specific probes (ITS1, ITS2, and 5' ETS), to detect all major precursor rRNAs. Note that the data show no significant differences in mature rRNA production or pre-rRNA processing between the parent and KD cells. $(D)$ RNA fluorescence in situ hybridization (FISH; green) combined with indirect immunofluorescence of Nopp140 (red/yellow; right panels) and DAPI DNA stain (blue; left panels) of a 1:1 mixture of parent and KD cells grown side by side. The detected RNAs are indicated above each pair of panels. Parent cells are recognized in the right panels by the nucleolar staining of Nopp140 (yellow because or overlap with green RNAs) and the knockdown cells are labeled (KD). Some CBs are indicated (arrowheads) and appear in red in the Nopp140 stain because they are devoid of (pre)-rRNAs. A combination of P1 and KD1a cells (28S rRNA and U3 snoRNA) and of P2 and KD2 cells (ITS2 and H/ACA snoRNAs) were used. To detect H/ACA snoRNAs, a combination of primers was used against E3, ACA8, ACA18, and ACA25. Scale bar, $10 \mu \mathrm{m}$. (E) Analysis of steadystate levels of select C/D and H/ACA RNAs by semi quantitative RT-PCR. RNAs tested and cell pairs analyzed are indicated above each lane. A simple number refers to that specific SNORD. The dashes at the left indicate the migration position of markers: $100 \mathrm{nt}$ if only one and 100 and $200 \mathrm{nt}$ if two. 
unsurprising considering the absence of any notable prerRNA processing and growth defects in Nopp140 KD cells.

To investigate the mechanism underlying the reduction in rRNA methylation at only a few residues, we used semiquantitative RT-PCR to interrogate the levels of some of the snoRNAs responsible for specifying the modifications. For this purpose, we isolated total RNA from parent and Nopp140 KD cells and used sno/scaRNA-specific primers in RT-PCR reactions. As previously established, scaRNAs displaced from CBs did not change in their abundance (e.g., hTR and scaRNA12/U89) (Bizarro et al. 2019), nor did that of the H/ACA snoRNA E3/SNORA63 (Fig. 5E). Similarly, 14 nucleolar box C/D snoRNAs did not vary in abundance between parent and KD cells irrespective of whether 2'-Omethylation of their target residue varied (SNORD98, SNORD127, SNORD50A, SNORD102, SNORD91B, and SNORD121A) or not (SNORD10, SNORD42A, SNOR D42B, SNORD4A, SNORD4B, SNORD15A, SNORD27, and 5SNORD5) (Fig. 5E). This despite the fact that for some targets, methylation was reduced by $>50 \%$ (SNO RD50A, SNORD102, SNORD91B, and SNORD121A) (Supplemental Table S2). Additionally, the levels of the box C/D snoRNA U3, which is involved in pre-rRNA processing, did not vary between parent and KD cells (Fig. 5E), nor did its localization (Fig. 5D). The results were independent of which pairs of cell lines were compared: P2 versus KD2 or P1 versus KD1a (Fig. 5E). Consequently, the reduced levels of 2'-O-methylation of certain nucleotides is not caused by fluctuations in levels of the snoRNPs that guide their modifications. This absence of correlation between snoRNA abundance and methylation levels observed here is consistent with previous studies using RMS (Krogh et al. 2016; Sharma et al. 2017). However, the low levels of Nopp140 in nucleoli leading to decompaction of the DFC apparently modified the access of snoRNPs to their targets affecting particularly those responsible for the methylation of normally already hypomodified residues. This may be similar to Nopp140 corralling those scaRNPs in CBs whose function there is required for snRNA modification, as compared with those that can also function in the nucleoplasm.

\section{Effects of Nopp140 KD on MRNA expression and pre- mRNA splicing fidelity}

The impact of Nopp140 KD on specific sites of 2'-O-methylation in several spliceosomal snRNAs provided us with the opportunity to test for the first time the function of these modifications in pre-mRNA splicing. For this purpose, total RNA-seq was performed on all cell lines. The reproducibility and robustness of our sequencing data were remarkable (e.g., Figs. 6E, 7B), given that total RNA from every cell line was isolated three times and as sequencing of the $\mathrm{P} 2$ and KD2 and of the P1, KD1a, and KD1b cell lines was performed $1 \mathrm{yr}$ apart and on different continents.

We first investigated differential expression of genes in the KD cells versus their parent cells. Whereas the number of up-regulated and down-regulated genes was similar for each of the three KD and parent pairs, the overall number of significantly differentially expressed genes (FDR 0.05 ) between KD1a and P1 was 5086 and that for KD1b and P1 was 3004 out of 13,778 genes analyzed (Fig. 6A, B). Similar numbers of differentially expressed genes were noted between $\mathrm{P} 2$ and KD2 cells (Fig. 6C). Limiting our analysis of differentially expressed genes to those common between all three pairs yielded 225 down-regulated and 192 up-regulated genes (Fig. 6D). Unsupervised clustering of these genes grouped together each triplicate RNA-seq, the parents $\mathrm{P} 1$ and $\mathrm{P} 2$, the KD cells KD1a, KD1b, and KD2 (Fig. 6E). Even the KD cells derived from the same parent cells, KD1a and KD1b, grouped together (Fig. 6E). Interrogating the differentially expressed genes according to gene ontology revealed almost exclusively genes associated with Nopp140 function. Specifically, down-regulated genes were highly enriched in genes involved in localization to CBs, nuclear bodies, nucleus, chromosomes, and telomeres (Fig. 6F). Apparently, in the absence of Nopp140, there is a reduced need for these codepleted genes. For unknown reasons, up-regulated genes showed a slight enrichment in genes involved in extracellular matrix and neuronal spine formation (Fig. 6G).

To assess the impact on alternative splicing of Nopp140 $\mathrm{KD}$ (i.e., the loss of 2'-O-methylation from several spliceosomal snRNAs), we used the rMATS algorithm for analysis of our RNA-seq data. Of the five different alternative splicing events, skipped exon (SE), retained intron (RI), alternative $3^{\prime}$ and $5^{\prime}$ splice sites (A3SS and A5SS), and mutually exclusive exons (MXE), SE events far outnumbered the others with $>50 \%$ in comparisons of all three parent and KD sets (Fig. 7A). This was in stark contrast to a comparison of the two parent cells where mutually exclusive exons with $44 \%$ far outnumbered skipped exons and the other splicing events (Fig. 7A). Therefore, reduced 2'-Omethylation of these snRNA residues preferentially affected one splicing pathway. However, only 153 SE events were common to all three sets indicating that general splicing remains unaltered (Fig. 7B). When analyzed by unsupervised clustering, each triplicate sample, the two parents, and the three KDs, all grouped together again highlighting the robustness of the data (Fig. 7B). Gene ontology analysis of the genes harboring the 153 SE events, did not reveal any significantly enriched GO terms. For further analysis and corroboration of the data, we focused on the RFXANK gene whose exon 5 was skipped about twice as often in the KDla cells compared with the parent. Specifically, exon 5 was included in $62 \% \pm 2 \%$ of mRNAs in parent P1 cells as shown on a sashimi plot (Fig. 7C). In contrast, the Nopp140 KD1a and KD1b cells included exon 5 only in $34 \% \pm 1 \%$ and $47 \% \pm 4 \%$ of mRNAs, respectively (Fig. 7C). Thus, also the degree of exon skipping parallels that of Nopp140 KD (Fig. 1AB) and that of the loss of snRNA 2'-O-methylation in those cells (Figs. 3-5). In other words, exon skipping is more pronounced in KDla than in KD1b cells (Fig. 7C) suggesting that these reproducible changes in alternative splicing are a direct consequence of reduced snRNA 2'-O-methylation. We corroborated the RNA-seq data by semiquantitative RT-PCR (Fig. 7D). Quantification of the results 
Downloaded from genesdev.cshlp.org on July 26, 2021 - Published by Cold Spring Harbor Laboratory Press

Splicing control by small nuclear RNA modification

Differentially expessed genes

A

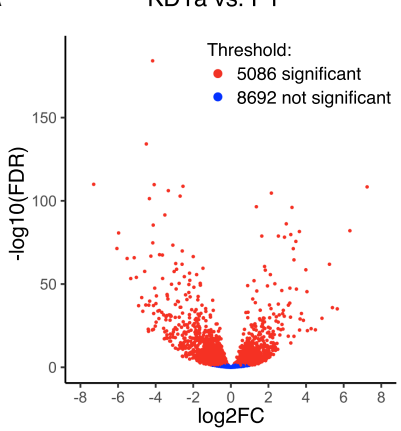

B

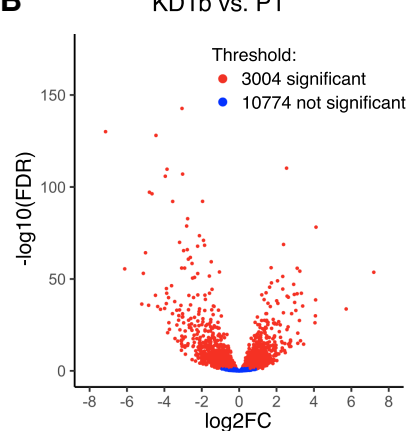

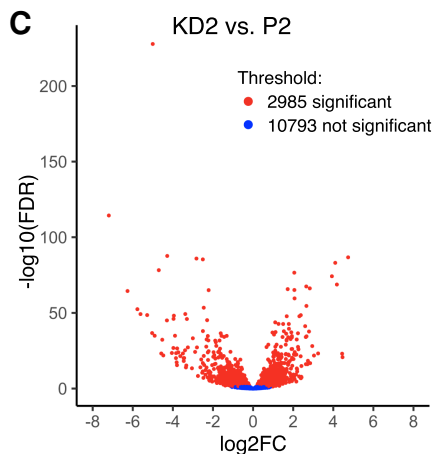

\section{Common differentially expessed genes}

D
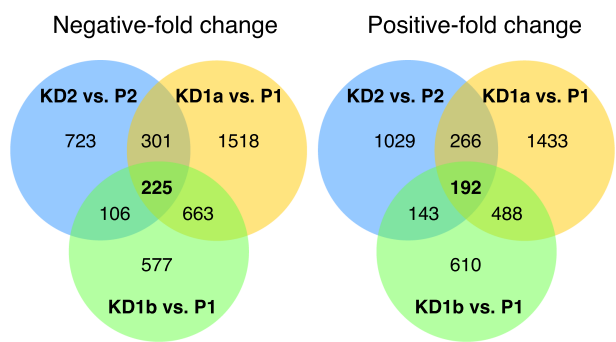

E

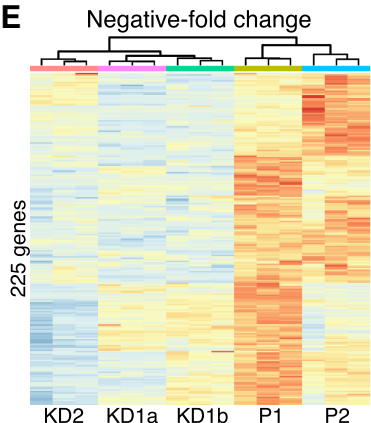

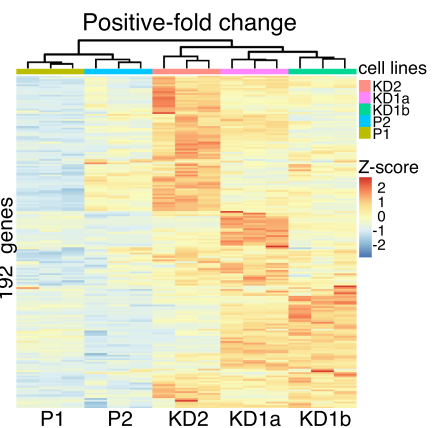

$\mathrm{P} 1 \quad \mathrm{P} 2 \quad \mathrm{KD} 2 \quad \mathrm{KD} 1 \mathrm{a} \quad \mathrm{KD} 1 \mathrm{~b}$

F

Negative-fold change

G Positive-fold change
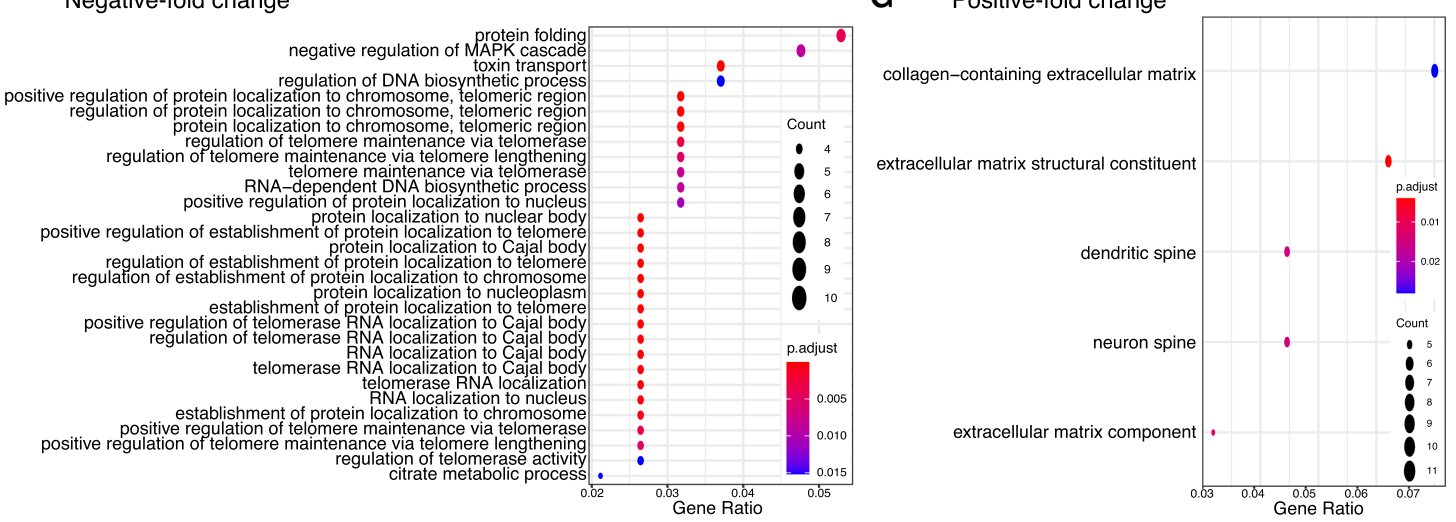

Figure 6. RNA-seq of total RNAs from Nopp140 parent and knockdown (KD) cells reveals few common differentially expressed genes. Volcano plots of genes differentially expressed between KD1a and P1 cells $(A)$, between KD1b and P1 cells $(B)$, and between KD2 and P2 cells $(C)$. $(D)$ Venn diagrams for common differentially expressed genes between the three pairs in $A-C$ for negative and positive fold change. (E) Heat map of $z$-scores for 225 common genes with negative and 192 common genes with positive fold change for all five cell lines. Note that despite the small differences, all biological triplicates and the parents and KD cells cluster together, even those KD cells originating from the same parent: KD1a and KD1b. $(F)$ Gene ontology $(\mathrm{GO})$ analysis of common differentially expressed genes with a negative fold change reveal mostly genes related to Nopp140 function. $(G)$ GO analysis of common differentially expressed genes with a positive fold change reveals only few genes. Gene ratio is the percentage of total differentially expressed genes within a given GO term.

expressed relative to $\mathrm{P} 1$ cells showed a loss of exon inclusion by $\sim 50 \%$ (Fig. 7E) mirroring the RNA-seq results (Fig. 7C). Importantly, exon 5 inclusion was restored in all three rescue cell lines (Fig. 7F,G), which paralleled the rescue of 2'-O-methylation of U1, U2, U5, and U12 snRNAs (Fig. 4C; Supplemental Table S1). These data thus strongly support the importance of snRNA modification for maintaining the fidelity of pre-mRNA splicing.

\section{Discussion}

In this study, we took advantage of our ability to separate the bulk of scaRNPs from their target snRNAs in CBs. This was achieved by KD of Nopp140 or expression of unphosphorylated Nopp140, resulting in CBs devoid of Nopp140 and scaRNPs. This approach allowed us to study the function of CBs in snRNA modification. We observed 
A

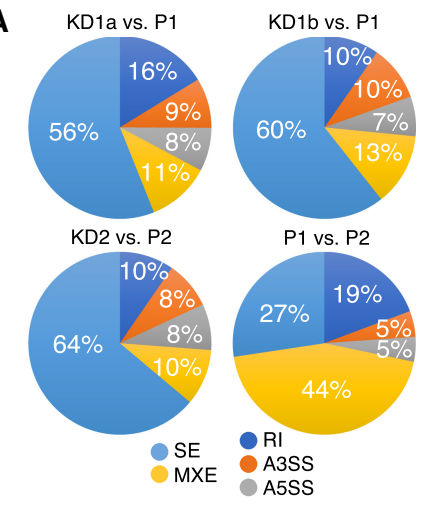

C

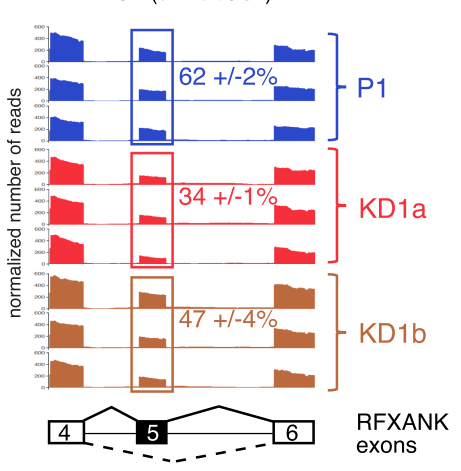

D

P1 KD1a KD1b P2 KD2

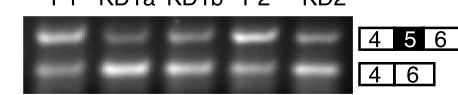

E

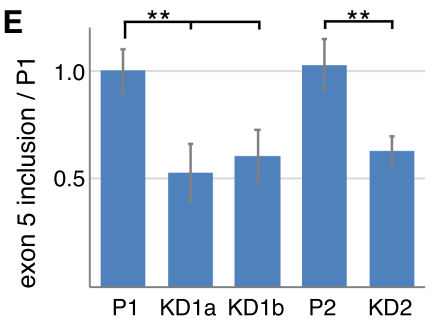

B

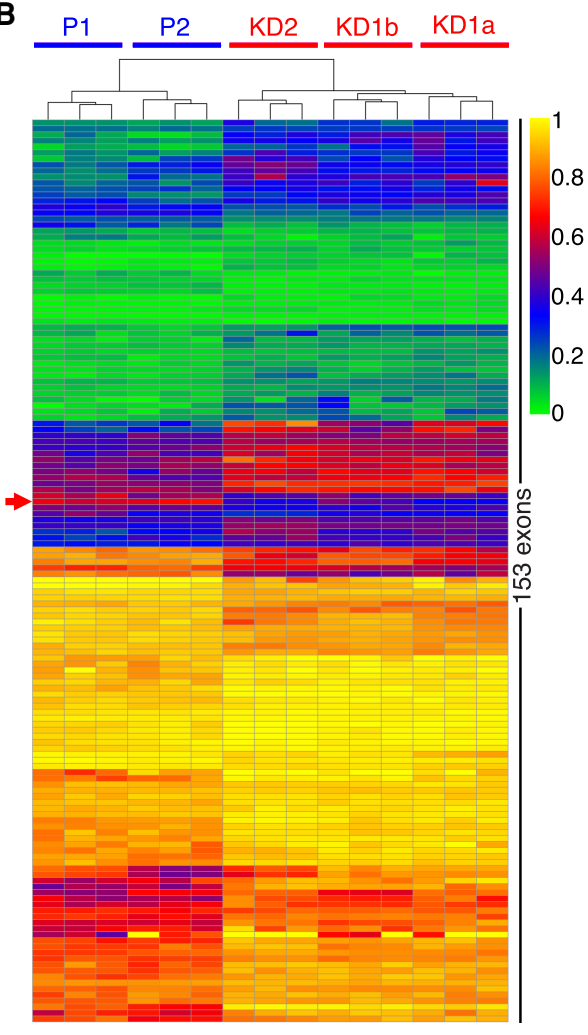

$\mathbf{F}$

P2 KD2 R2b R2a R2c
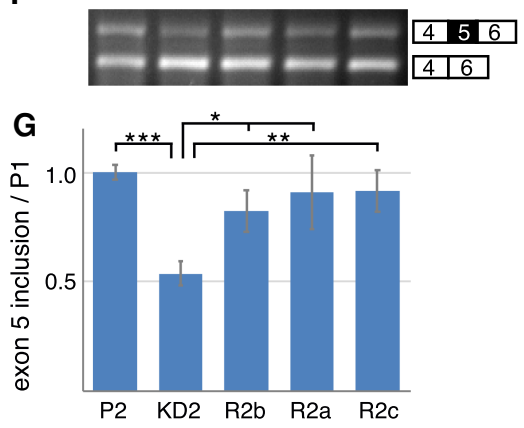

Figure 7. Analysis of RNA-seq data for alternative splicing events using the rMATS algorithm shows small but significant changes after Nopp140 KD. (A) Analysis of splicing events of Nopp140 KD cells relative to their corresponding parent cells contrasted to those normally occurring in parent versus parent cells. The analyzed events expressed in the percentage pie chart are skipped exons (SE), mutually exclusive exons (MXE), retained introns (RI), alternative $3^{\prime}$ splice site (A3SS), and alternative $5^{\prime}$ splice site (A5SS). (B) Heat map of 153 alternatively spliced exons in all cell lines arranged through unsupervised clustering. Note the remarkable clustering of all triplicates and the parent versus KD cells. The RFXANK exon 5 is indicated (red arrow). (C) Sashimi plot of the sequence traces spanning the region of RFXANK exons 4-6 (schematically depicted below) from triplicate analysis. For direct comparison, the reads are normalized and the mean inclusion levels $( \pm \mathrm{SD})$ of exon 5 (boxed) are indicated. Note the larger difference of exon 5 inclusion of KDla over KDlb cells relative to the parent P1 mirroring the degree of Nopp140 $\mathrm{KD}$ in those cells. Also note the small SD values. The plots were generated using ggsashimi (https ://github.com/guigolab/ggsashimi). (D) Semiquantitative RT-PCR of the RFXANK exons 46 separated on agarose gels and stained by ethidium bromide confirm the deep sequencing rMATS results for exon 5 inclusion. (E) Quantification of quadruplicate results in $D$. Unpaired $t$ tests identify significant differences. $\left({ }^{* *}\right) P<$ 0.005. $(F)$ As in $D$ but including RNA from all three rescue cell lines documenting the restoration of exon 5 inclusion in Nopp140-re-expressing cell lines. $(G)$ Quantification of triplicate results in $F$. Unpaired $t$-tests identify significant differences. $\left.\left({ }^{*}\right) P<0.05,\left({ }^{* *}\right) P<0.005,(* *)\right) P<$ 0.0005 . that in Nopp140 KD cells, snRNA pseudouridylation proceeds normally but most sites of 2'-O-methylation were impaired. Genome-wide analysis shows an overall switch in pre-mRNA splicing events from mutual exclusion of exons to exon skipping and a reproducible change in alternate splice site usage. All these effects are due to Nopp140 $\mathrm{KD}$ as their degree parallels that of KD levels and because Nopp140 re-expression rescues all effects. Two main conclusions can be drawn from these observations, most snRNA 2'-O-methylation occurs in $\mathrm{CBs}$ and is required to maintain splicing fidelity.

A surprising finding was the specific effect on 2'-Omethylation but not on pseudouridylation, at least not at the interrogated sites. These data support the notion that CBs are not obligatory sites for pseudouridylation of snRNAs but that this modification can also occur in the nucleoplasm. In fact, in Drosophila and HeLa cells,
snRNAs are efficiently modified even in the absence of CBs (Deryusheva and Gall 2009, 2013; Deryusheva et al. 2012). In our cell system, coilin-positive CBs with snRNPs persist even without scaRNPs and Nopp140 (Bizarro et al. 2019|. Therefore, the modifications still occurring appear essential for pre-mRNA splicing. In particular, pseudouridines in U2 snRNA have long been identified as important for in vitro reconstituted splicing reaction in yeast extracts (McPheeters et al. 1989). Pseudouridines of yeast U2 snRNA are further important for stimulating the ATPase activity of Prp5 during spliceosome assembly (Wu et al. 2016). Finally, pseudouridines in the branch site recognition region of $\mathrm{U} 2 \mathrm{snRNA}$ and in general are required for pre-mRNA splicing and snRNP biogenesis in vivo and in vitro (Yu et al. 1998; Zhao and Yu 2004, 2007).

In contrast, most 2'-O-methyl groups of snRNAs U1, $\mathrm{U} 2$, U5, and U12 are lost in Nopp140 KD cells allowing 
investigation of their role in pre-mRNA splicing. As Nopp140 KD cells proliferate at identical rates as their parent cells (Bizarro et al. 2019), 2'-O-methylations of snRNAs appear less important than pseudouridines for overall splicing. This is supported by the fact that in budding yeast, snRNAs are pseudouridylated, but 2'-O-methylation has not been observed (Massenet et al. 1998). Nevertheless, we show that reduced 2'-O-methylation of snRNAs compromises alternative splicing. Apparently, 2 -O-methylation in the nucleoplasm is not as efficient in the absence of scaRNPs from CBs arguing that CBs function by enhancing the activity of scaRNPs on snRNAs by bringing them together following the law of mass action. Apparently, an opposite function of CBs is in place for the telomerase RNP, in which case CBs function to sequester telomerase from its nucleoplasmic substrates, the telomeres (Bizarro et al. 2019)

Interestingly, two 2'-O-methyl groups within U2 snRNA are not impacted by Nopp140 KD, Gm12 and Gm25. In the active spliceosome, those 2'-O-methylated residues lie between helix $\mathrm{Ia}$ and $\mathrm{Ib}$, and right adjacent to helix II formed between U2 and U6 snRNAs, perhaps pointing to an especially important role in splicing (Zhang et al. 2017; Townsend et al. 2020). In fact, U2-Gm12 was one of four 2'-O-methylated residues within the first 20 $\mathrm{nt}$ of HeLa U2-snRNA that was required for in vitro splicing (Dönmez et al. 2004). U2-Gm12 and U2-Gm25 form the base of stem loop I (SL1) and of the branchpoint-interacting stem loop (BSL), respectively, in the 17S U2 snRNP (Zhang et al. 2020). However, they are both located in the center of SL1 of the extended U2 conformation in the active spliceosome. Apparently, the modifications of these two guanosines exhibit a more basic function in splicing than the remaining ones of U2 and of all other snRNAs. Similarly, pseudouridines cannot be lost from snRNAs, although to determine whether this is true for all pseudouridines will require a more detailed genome-wide approach, perhaps using the recently developed HydraPsiSeq (Marchand et al. 2020).

Although we argue that pseudouridylation of snRNAs in general and 2'-O-methylation at U2-Gm 12 and Gm25 is too important to be lost and therefore mediated by scaRNPs outside CBs, it is also possible that redundant enzymes are responsible for these activities. For example, the stand-alone pseudouridine synthases Pus7 and Pus1 are capable of pseudouridylating U34 and U43 of mammalian U2 snRNA, respectively (Morais et al. 2021). However, these enzymes potentially account for only a few of the many pseudouridines in mammalian snRNAs and it is unknown whether such mechanisms exist for the 2'-Omethylation of mammalian snRNAs.

Methylation of 2'-O-ribose of U6 snRNA remained unaffected in the Nopp140 KD cells. This is not surprising given that its modification occurs in nucleoli and not CBs (Ganot et al. 1999). In fact, guiding the 2'-O-methylation of U6 snRNA follows an altogether different pathway than that for all other snRNAs. The La related protein 7 (LARP7) is responsible for bringing together U6 snRNA and a specific subset of C/D snoRNAs required for its modification (Hasler et al. 2020). Thus, LARP7 may func- tion for U6 in the nucleoplasm as Nopp140 does for all other snRNAs in CBs.

While overall 2'-O-methylation of snRNAs is clearly reduced in Nopp140 KD cells that of rRNA is affected to a much lesser extent. Only $13 \%$ and $18 \%$ of 2 -O-methylated residues of $18 \mathrm{~S}$ and $28 \mathrm{~S}$ rRNA, respectively, are impacted by Nopp140 KD. Importantly, all but three of the sites affected by Nopp140 KD are normally not fully 2'-Omethylated in cellular rRNA. Apparently, these hypomodified residues are generally less important for proper ribosome biogenesis and function and thus more susceptible to minor changes in their cellular environs. This conclusion seems similar to the detrimental effect on general pre-mRNA splicing by the loss of snRNA pseudouridylation but not the reduction of snRNA 2'-O-methylation. Regardless, as in the case of the two guanosines of U2 snRNA (Gm12 and Gm25) that fail to lose their 2'-Omethyl groups, the loss at those three nucleotides (18SUm428, 28S-Gm4370, and 28S-Cm4456) normally modified to the full extent may be the ones to look at for consequences. Given that the Nopp140 KD cells produce ribosomes normally and exhibit the same growth rate as their parent cells, it is not surprising that no obvious function can be assigned to those three nucleotides, except that they are positioned near the subunit interface.

Our data contrast the consequences of a complete and lethal deletion of two Nopp140 homologs in Drosophila (He et al. 2015). In that case, 2'-O-methylation in two regions of ribosomal RNA was suppressed by approximately fourfold. However, Drosophila Nopp140 differs significantly from its mammalian counterpart as its two splice variants produce two different proteins, one more closely related to nucleolin and the other to Nopp140 (Waggener and DiMario 2002). Therefore, assignment of the observed effects is complex. Importantly, snRNA modification was not assessed in that case. Nevertheless, effects of human Nopp140 KD, other than the established scaRNP displacement from CBs, also need to be considered. Though there is no evidence for Nopp140 itself having any methyltransferase activity, it could play a role in efficient modification at specific sites by allowing access to other chaperones and helicases.

The low impact on rRNA but high impact on snRNA modification by Nopp140 KD may be further explained by the presence of residual Nopp140 in nucleoli but not CBs. This was particularly surprising given that in parent cells Nopp140 fluorescence intensities of CBs matched those of nucleolar DFCs indicating a specific loss of Nopp140 from CBs but only a reduction in nucleoli. The extreme transcription rate of rDNA and the concomitant near normal rRNA modification in regularly growing Nopp140 KD cells draws a typical accumulation of snoRNPs in DFCs, which may be responsible for the visible Nopp140 accumulation even in KD cells. In contrast, as outlined above, most scaRNPs are displaced from CBs into the nucleoplasm in $\mathrm{KD}$ cells where residual Nopp140 is more dispersed and less detectable. In other words, nucleoli are obligate cellular organelles whereas CBs are not but may simply enhance activity by coconcentration of scaRNP enzymes and their snRNA 
substrates. This is supported by ample data of cells functioning and splicing normally even in the absence of CBs (Spector et al. 1992; Deryusheva and Gall 2009, 2013; Deryusheva et al. 2012).

The robustness of the overall RNA-seq results is remarkable and with it that of pre-mRNA splicing analysis using the rMATS algorithm. In particular, unsupervised clustering not only aligns the biological triplicates of each cell line but also clusters the parent and KD cells together, even the KD cells derived from the same parent cells. This despite the fact that library preparation and sequencing of the two parent-KD cell pairs was performed a year apart and on different continents. Although overall splicing was minimally affected, there were some obvious differences between parent and KD cells. There was a clear shift in preference for splicing events when parent cells were compared with $\mathrm{KD}$ versus each other. In particular, comparison with KD cells showed a strong preference for exon skipping events. Overall, 153 alternatively spliced exons were identified consistently in all three KD cells highlighting the effect of reduced snRNA 2'-O-methylation on pre-mRNA splicing. Importantly, the degree of effect paralleled that of reduction in snRNA 2'-O-methylation and that of Nopp140 KD levels clearly linking these events. This was further corroborated by the rescue of all effects by re-expression of Nopp140. Mechanistically, the entire chain of events depends on the Nopp140-mediated concentration of scaRNPs (and Nopp140) in CBs, which we show relies on the extreme level of phosphorylation of Nopp140.

Nopp140 phosphorylation at $\sim 80$ serines is mediated by $\mathrm{CK} 2$ and is required for accumulation in CBs, but not nucleoli. Apparently, phosphorylation of Nopp140 is not sufficient for its interactions with snoRNPs in nucleoli but is for those with scaRNPs in CBs. Therefore, inhibition of Nopp140 phosphorylation specifically inhibits scaRNP localization to CBs and with it most 2'-O-methylation of snRNAs resulting in altered splicing fidelity. These phosphorylation-specific effects seem surprising given that normally dephosphorylation of Nopp140 is not observed and consequently only newly synthesized Nopp140 appears a target for CK2. Nevertheless, these effects need to be taken into consideration in therapy with the CK2 inhibitor CX-4945 (silmitasertib) for cholangiocarcinoma, hematological malignancies, and COVID-19.

\section{Materials and methods}

Plasmids

Plasmids used to generate the stable rescue clones were pNK65 and pJB9 expressing HA-Nopp140-GFP under a CMV promoter or UBC promoter, respectively. Transient rescue during CKII inhibition was performed using plasmid pJB8 expressing HANopp140 under CMV promoter (Bizarro et al. 2019).

Cell culture, transfection, and genome engineering

HeLa cells and the various clones were cultured in DMEM (Gibco) and 10\% fetal bovine serum (Atlanta Biologicals) at $37^{\circ} \mathrm{C}$ under $5 \% \mathrm{CO}_{2}$ in air. Nopp140 KD clones were generated as described using CRISPR/Cas9 technology (Bizarro et al. 2019). RNA-seq in this study confirmed proper targeting of the sgRNAs with most reads carrying mutations at the targeted sites. Transfections were performed with Lipofectamine 3000 (Thermo Fisher Scientific) following the manufacturer's protocol. CK2 inhibition was performed on HeLa cells or on Nopp140 KDla cells transfected with pJB8 plasmid $6 \mathrm{~h}$ after addition of CX-4945 (Selleckchem) at $10 \mu \mathrm{M}$ in DMEM, $10 \%$ FBS for 1,2 , or $3 \mathrm{~d}$ before analysis by Western blotting and indirect immunofluorescence. Stable rescues were generated in the Nopp 140 KD2 cells by transfection with pJB9 or pNK65 plasmids. Transfected cells were treated with G418 (1 g/mL final; Corning) for $2 \mathrm{mo}$. Single clones were obtained by limited dilution and tested for Nopp140 re-expression by indirect immunofluorescence and Western blotting. Transfection with pJB9 yielded clones R2a and R2b and that with pNK65 yielded clone R2c. Nopp140 expression in the rescue clones remained stable after several months in culture.

\section{Antibodies}

Antibodies (dilutions in parentheses) for Western blotting (WB) or indirect immunofluorescence (IF) were as follows: anti-Nopp140 rabbit serum (RS8 at 1:5000 for WB and 1:1000 for IF) (Kittur et al. 2007), mouse monoclonal anti-NAP57 immunoglobulin G (IgG) (H3 at 1:500 for IF; Santa Cruz Biotechnology), mouse monoclonal anti- $\beta$-actin (AC-15 at 1:1000 for WB; Santa Cruz Biotechnologies), mouse anti- $\gamma$-tubulin ascites fluid (GTU-88 at 1:5000 for WB; Sigma), DyLight488 goat antimouse IgG (1:500 for IF) and rhodamine (TRITC) goat antirabbit IgG (1:500 for IF; both Jackson ImmunoResearch), Alexa Fluor 680 goat antirabbit IgG (1:10,000 for WB; Thermo Fisher Scientific), and IRDyeTM 800 goat antimouse IgG (1:10,000 for WB; Rockland Immunochemicals).

\section{Western blotting}

For each experiment, proteins from the same number of cells per condition were extracted into SDS-sample buffer $(0.5 \mathrm{M}$ Tris at pH 6.8, 12\% SDS, $0.05 \%$ bromophenol blue). The lysates were tip-sonicated and total proteins loaded (100,000 cell equivalents), separated on $9 \%$ SDS-PAGE, and transferred to nitrocellulose membrane. Transfer efficiency was confirmed by Ponceau red staining, and membranes were blocked in blocking buffer (Trisbuffered saline, $0.1 \%$ Tween, $2.5 \%$ nonfat dry milk) for $30 \mathrm{~min}$ before incubation with primary antibodies diluted in blocking buffer overnight at $4^{\circ} \mathrm{C}$. After three washes in blocking buffer, membranes were incubated with appropriate secondary antibodies diluted in blocking buffer for $1 \mathrm{~h}$ at room temperature in the dark. After three washes in blocking buffer, membranes were scanned on an Odyssey 9120 imaging system (LI-COR Biosciences), and protein bands were quantified using Image Studio Lite (LICOR Biosciences) and analyzed with Microsoft Excel and GraphPad Prism software.

\section{Indirect immunofluorescence}

Cells grown on coverslips were fixed in $4 \%$ paraformaldehyde (PFA) in phosphate-buffered saline (PBS) for $20 \mathrm{~min}$, permeabilized with $1 \%$ Triton X-100 in PBS for 5 min, and blocked with $1 \%$ powdered milk in PBS (IF blocking buffer) for $15 \mathrm{~min}$. The cells were then incubated for $2 \mathrm{~h}$ with primary antibodies in IF blocking buffer, washed, and incubated for $1 \mathrm{~h}$ with secondary antibodies in IF blocking buffer in the dark. This was followed by washing and DNA staining with 4',6-diamidino-2-phenylidone (DAPI; $1 \mu \mathrm{g} / \mathrm{mL}$ in PBS). Coverslips were mounted on glass slides using ProLong Diamond antifade mount (Thermo Fisher 
Scientific) and observed using a Zeiss Axio Observer Z1 fluorescence microscope $(63 \times$ objective, $1.4 \mathrm{NA})$ with filter sets $34-$ DAPI (Zeiss 000000-1031-334), 10-AF488 (Zeiss 488010-9901000), 43HE-DsRED (Zeiss 489043-9901-000), and 50-Cy5 (Zeiss 488050-9901-000). Z-stack images in 200-nm steps were acquired with a Zeiss AxioCam MRm camera using Axiovision software (Zeiss). Maximum projections were generated using ImageJ (National Institutes of Health). Quantification of Nopp140 protein signals in nucleoli and Cajal bodies was done using ImageJ with the help of macros (available upon request). Briefly, NAP57 images were used to locate the nucleoli and Cajal bodies around which masks were generated. The DAPI images were used to establish nuclear masks. These masks were applied to the Nopp140 images to determine their signal intensity in the organelles per nucleus. Background was subtracted individually for each nucleolus and Cajal body and was defined as the pixel with the lowest signal in a 50-pixel circumference of the mask using an ImageJ function. Images for figures were cropped and adjusted using Photoshop CC (Adobe). To compare parent, KD, and rescue cell images, all images within the same panels and of the same antigens were acquired and adjusted identically.

\section{RNA fluorescent in situ hybridization (FISH)}

Cells on coverslips were fixed with PBS, 4\% PFA, permeabilized with PBS, $1 \%$ Triton X-100, washed with $2 \times$ SSC, and $40 \%$ formamide. RNAs were stained for $4 \mathrm{~h}$ at $37^{\circ} \mathrm{C}$ in the dark using 32-50mer probes (Supplemental Table S3) synthesized and internally labeled with Cy3 as described (Chartrand et al. 2000). Hybridization with denatured probes $(2 \mathrm{ng} / \mu \mathrm{L})$ was performed for 3 $\mathrm{h}$ at $37^{\circ} \mathrm{C}$ in $2 \times \mathrm{SSC}, 40 \%$ formamide, $50 \mathrm{ng} / \mathrm{\mu L}$ ssDNA/tRNA, and $3.5 \mu \mathrm{g} / \mu \mathrm{L}$ BSA. Cells were washed extensively with $2 \times$ SSC, $40 \%$ formamide at $37^{\circ} \mathrm{C}$, then with PBS, before fixation in PBS and $4 \%$ PFA, and incubation in blocking buffer (PBS, $1 \%$ dry milk). Cells were then incubated with Nopp140 antibodies (RS8 at $1: 1000$ ) in blocking buffer for $2 \mathrm{~h}$ followed by secondary antibodies (rabbit Alexa 488 at 1:500) in blocking buffer for $1 \mathrm{~h}$ in the dark. After washes in blocking buffer and DAPI staining, the coverslips were mounted using ProLong Diamond. The samples were observed using an Olympus IX81 epifluorescence microscope with a $60 \times$ objective, $1.4 \mathrm{NA}$, oil immersion objective. Z-stack images in 200-nm steps were acquired with a Sensicam QE cooled CCD camera using IP Lab 4.0.8 software and processed using Adobe Photoshop.

\section{Total RNA extraction and sequencing}

RNA from the different cell lines was extracted using $500 \mu \mathrm{L}$ of TRIzol reagent (Ambion) directly on 10-cm dishes (cell confluency $\sim 80 \%, \sim 1,000,000$ cells). Lysed cells in TRIzol were scraped into tubes, extracted twice with chloroform, the RNA was precipitated with $0.7 \mathrm{vol}$ of isopropanol after addition of $20 \mu \mathrm{g}$ of glycogen, and resuspended in UltraPure distilled water. RNA concentration and quality were determined by Nanodrop (ratio 260/230 and 260/280 above 1.8) and Agilent 2100 BioAnalyzer (RIN above 8). Total RNA was used for Northern blot analysis, RiboMethSeq, RT-PCR, and RNase H analysis. For deep sequencing, total RNA was prepared from three separate dishes for each sample and shipped to Novogene Corporation, Inc., for cDNA library preparation (250 to 300 -bp inserts) and Illumina sequencing (PE150). The RNA was prepared and sequenced 1 yr apart in the USA and in China in two batches: one for the P1, KD1a, and KD1b cells, and one for the P2 and KD2 cells. All data have been deposited in the GEO repository under the accession number GSE173171.

\section{RNA-seq data analysis}

Raw Fastq files were obtained from Novogene and were checked for quality of reads with FastQC (version 0.11.4). Raw reads were aligned with the splice aware aligner STAR (version 2.4.2a) (Dobin et al. 2013). Cufflinks (version 2.2.1) was used to generate FPKM expression values (Trapnell et al. 2010). The featureCounts from Subread package (1.5.0-p1) was used to count the number of raw fragments associated with each gene (Liao et al. 2014). Differential gene expression analysis was performed with the help of the Bioconductor package DESeq2 (Love et al. 2014). Gene ontology (GO) analysis of common differentially expressed genes was performed using clusterProfiler (Yu et al. 2012). Multivariate analysis of transcript splicing with replicates (rMATS, version 3.2.5) was used to detect differential splicing events. Significant events with FDR $\leq 0.05$ are reported (Shen et al. 2014).

\section{Pre-rRNA processing analysis by Northern blotting}

Total RNA was extracted with the TRIzol reagent (Ambion) according to the manufacturer's instructions. Three micrograms of total RNA was separated on $1.2 \%$ denaturing agarose gel, transferred to a nylon membrane, and hybridized with 32P-labeled oligonucleotide probes specific to all major pre-rRNAs, as described in Tafforeau et al. (2013) and Sharma et al. (2015). The membrane was exposed to Fuji imaging plates (Fujifilm). The signals were acquired with a phosphorimager (Fujifilm FLA-7000) and quantified with the native Multi Gauge software. Probe sequences are in Supplemental Table S4.

\section{Analysis of 2'-O-methylation levels by RiboMethSeq}

RMS was performed exactly as described in Marchand et al. (2016). For each reaction, 150 ng of total RNA was used. The samples were sequenced at the ULB-BRIGHTcore facility (Brussels Interuniversity Genomics High-Throughput Core) on Illumina Novaseq 6000 as paired-end runs (100-nt read length). In average, 25 million reads were sequenced (Supplemental Table S5).

Adapter sequences were removed using Trimmomatic 10.36 ; leading:30 trailing:30 slidingwindow:4:15 minlen:17 avgqual:30) and reads in forward direction were mapped to an artificial genome containing ribosomal RNA sequences using bowtie2 (2.3.3.1; sensitive). Mapped reads were analyzed using the R package version 1.2.0 RNAmodR.RiboMethSeq(https://bioconductor .org/packages/release/bioc/html/RNAmodR.RiboMethSeq .html) and the Score C/RiboMethScore was used as a measurement for 2'-O methylation. For the analysis of methylation levels on positions known to be methylated, data from the snoRNAdb were used (Lestrade and Weber 2006) and updated to revised rRNA sequence coordinates based on NCBI accession NR_046235.3, which are available from the EpiTxDb R package version 1.0.0 (http://www.bioconductor.org/packages/release/ bioc/html/EpiTxDb.html).

Fluorescent primer extension analysis of RNA modification

To analyze 2'-O-methylation and pseudouridylation patterns of human U2 snRNA we used a fluorescent primer extension method as described (Deryusheva and Gall 2009; Deryusheva et al. 2012). In brief, to detect 2'-O-methylated positions reverse transcription reactions were performed at very low concentration of dNTP. It has been shown previously that different reverse transcription enzymes have different termination efficiency at 2'-O-methylated positions (Deryusheva et al. 2012). We used EpiScript RT (Epicentre) to assess U2-Am30 semiquantitatively, and AMV-RT (New England Biolabs) to assess U2-Um47. To 
map pseudouridines, RNA samples were treated with CMC (N-cyclohexyl-N9-[2-morpholinoethyl] carbodiimide metho-ptoluene sulfonate) followed by incubation in sodium carbonate buffer ( $\mathrm{pH}$ 10.4). Reverse transcription was done at $0.5 \mathrm{mM}$ dNTP using EpiScript RT.

Each RNA sample was tested in two to three replicates. Sets of RNAs from parental and Nopp140KD lines were always treated simultaneously and separated on capillary columns in parallel using serial dilutions. GeneMapper 5 software (Applied Biosystems) was used to visualize and analyze the data.

\section{RT-PCR-based RNA modification analysis}

Another RT-based method to assay RNA modification levels semiquantitatively uses the same principles as described above but instead of separation of fluorescently labeled ssDNA fragments on capillary columns it involves qPCR with two sets of oligos. One set contains a forward oligo that anneals downstream from modified positions, and the other set contains a different forward oligo that anneals upstream of the modified positions (Dong et al. 2012). Oligonucleotides used to assess modifications in U2 snRNA and 18S rRNA are depicted in Figures 3E and 4C, respectively. EpiScript RT was used on CMC treated and untreated U2 snRNA and AMV-RT on 18S rRNA.

\section{Site-directed cleavage of RNA by RNase $H$}

To test and quantify 2'-O-methylation levels of selected positions in U2, U5, U6, and U12 snRNAs, we used a technique that uses RNase $\mathrm{H}$ site-specific cleavage of RNA directed by RNA-DNA chimeric oligonucleotides. The method was developed in Joan Steitz's laboratory and is based on the ability of 2'-O-methylated residues to protect RNA in RNA-DNA hybrid from RNase H digestion (Yu et al. 1997). It is known that in this assay the position of cleavage depends on the source of enzyme (Lapham et al. 1997). We tested RNase H (New England Biolabs) on control unmodified and single modified RNA oligos and designed experimental chimeric oligos accordingly. The chimeric oligos used were as follows: U2-Gm25 $\left(\mathrm{rU}_{\mathrm{m}} \mathrm{rG}_{\mathrm{m}} \mathrm{rA}_{\mathrm{m}} \mathrm{rU}_{\mathrm{m}}\right.$ dCdTdTdArG $\mathrm{G}_{\mathrm{m}} \mathrm{rC}_{\mathrm{m}}$ $\left.\mathrm{rC}_{\mathrm{m}} \mathrm{rA}_{\mathrm{m}} \mathrm{rA}_{\mathrm{m}} \mathrm{rA}_{\mathrm{m}} \mathrm{rA}_{\mathrm{m}} \mathrm{rG}_{\mathrm{m}}\right)$, U2-Cm40 ( $\mathrm{rG}_{\mathrm{m}} \mathrm{rA}_{\mathrm{m}} \mathrm{rA}_{\mathrm{m}} \mathrm{rC}_{\mathrm{m}} \mathrm{rA}_{\mathrm{m}} \mathbf{d G d A}$ dTdArC $\left.\mathrm{m}_{\mathrm{m}} \mathrm{U}_{\mathrm{m}} \mathrm{rA}_{\mathrm{m}} \mathrm{rC}_{\mathrm{m}} \mathrm{rA}_{\mathrm{m}} \mathrm{rC}_{\mathrm{m}} \mathrm{rU}_{\mathrm{m}} \mathrm{rU} \mathrm{U}_{\mathrm{m}}\right)$, U5-Um41 ( $\mathrm{rG}_{\mathrm{m}} \mathrm{rU}_{\mathrm{m}} \mathrm{rA}$ $\mathrm{rA}_{\mathrm{m}}$ dAdAdGdGrC $\left.\mathrm{rG}_{\mathrm{m}} \mathrm{rA}_{\mathrm{m}} \mathrm{rA}_{\mathrm{m}} \mathrm{rA}_{\mathrm{m}} \mathrm{rG}_{\mathrm{m}} \mathrm{rA}_{\mathrm{m}}\right)$, U6-Cm77 $\left(\mathrm{rU}_{\mathrm{m}} \mathrm{r}\right.$ $\mathrm{G}_{\mathrm{m}} \mathrm{rC}_{\mathrm{m}} \mathrm{rG}_{\mathrm{m}} \mathrm{rU}_{\mathrm{m}}$ dGdTdCdArU $\mathrm{m}_{\mathrm{m}} \mathrm{rC}_{\mathrm{m}} \mathrm{rC}_{\mathrm{m}} \mathrm{rU}_{\mathrm{m}} \mathrm{rU}_{\mathrm{m}} \mathrm{rG}_{\mathrm{m}} \mathrm{rC}_{\mathrm{m}}$, and U12$\mathrm{Gm} 22\left(\mathrm{rU}_{\mathrm{m}} \mathrm{rU}_{\mathrm{m}} \mathrm{rU}_{\mathrm{m}} \mathrm{rU}_{\mathrm{m}} \mathrm{rC}_{\mathrm{m}}\right.$ dCdTdTdArC $\left.\mathrm{m}_{\mathrm{m}} \mathrm{rU}_{\mathrm{m}} \mathrm{rC}_{\mathrm{m}} \mathrm{rA}_{\mathrm{m}} \mathrm{rU}_{\mathrm{m}}\right)$.

In 4-nt DNA regions (bold), the position that pairs with a 2'-Omethylated residue in tested RNA is underlined. RNA residues in the chimeras are 2'-O-methylated to stabilize oligos and increase specificity $\left(\mathrm{rN}_{\mathrm{m}}\right)$. Test RNAs in the amount of 3-5 $\mu$ g were mixed with $20-50$ pmol of a chimeric oligo in $15 \mu \mathrm{L}$ of RNase $\mathrm{H}$ reaction buffer. The mixture was heated for $5-10 \mathrm{~min}$ at $65^{\circ} \mathrm{C}$ and annealed for $10 \mathrm{~min}$ at $37^{\circ} \mathrm{C}$. Then $2-5 \mathrm{U}$ of RNase $\mathrm{H}$ (New England Biolabs) in $5 \mu \mathrm{L}$ of $1 \times$ RNase $\mathrm{H}$ buffer were added to the annealed RNA chimeric oligo mixture and the RNase $\mathrm{H}$ cleavage reaction was performed for $1 \mathrm{~h}$ at $37^{\circ} \mathrm{C}$. In vitro transcribed (unmodified) snRNAs were used as controls for RNase $\mathrm{H}$ digestion efficiency; RNA samples incubated without chimeric oligos or RNase $\mathrm{H}$ served as additional controls. The digested RNAs were separated on $8 \%$ polyacrylamide $/ 8 \mathrm{M}$ urea gels, transferred onto a nylon membrane (Zeta Probe, Bio-Rad) and probed with digoxigenin (Dig)-labeled DNA fragments corresponding to human U2 (nucleotides 61-3' end), U5 (nucleotides 1-41), U6 (nucleotides 1-77), and U12 (nucleotides 23-3' end). Dig was detected using antiDig antibody conjugated with alkali phosphatase and CDP-Star substrate (Roche) according to manufacturer's protocols. Li-Cor
Odyssey Fc imaging system and Image Studio software were used to visualize and quantify results. Each RNA sample was assayed in two to three replicates; each replicate was split to run on two separate gels and to probe independently for reproducibility control.

\section{RT-PCR}

Semiquantitative RT-PCR were performed on $1000 \mathrm{ng}$ of DNase RQ1 (Promega) treated total RNA using SuperScript III one-step RT-PCR system (Thermo Fisher Scientific) following the manufacturer's instructions. DNA fragments were separated on $4 \%$ for PCR products $<100 \mathrm{bp}$ or $2 \%$ agarose gels and bands quantified using Image Studio Lite (LI-COR Biosciences). Primers (Thermo Fisher Scientific) used for RT-PCR are described in Supplemental Table S6.

\section{Electron microscopy}

Monolayers of cells were fixed with $2.5 \%$ glutaraldehyde in $0.1 \mathrm{M}$ sodium cacodylate buffer, postfixed with $1 \%$ osmium tetroxide followed by $2 \%$ uranyl acetate, and dehydrated through a graded series of ethanol, and the cells were lifted from the monolayer with propylene oxide and embedded as a loose pellet in LX112 resin (LADD Research Industries) in Eppendorf tubes. Ultrathin sections were cut on a Leica Ultracut UC7, stained with uranyl acetate followed by lead citrate, viewed on a JEOL 1400 Plus transmission electron microscope at $80 \mathrm{kV}$, and images electronically acquired. CB granule size and number was assessed using ImageJ (National Institutes of Health).

\section{Competing interest statement}

The authors declare no competing interests.

\section{Acknowledgments}

The microscopes used are maintained by the Einstein Analytical Imaging Facility (AIF), which is partially supported by the National Institutes of Health (NIH)-funded Einstein Cancer Center (P30CA013330). For RNA FISH, access to the DS1 microscope was generously provided by Rob Singer. We are grateful to Louis Hodgson for the actin antibody. Leslie Gunther-Cummins and Xheni Nishku (AIF) prepared and imaged the samples on an electron microscope supported by an NIH-funded Shared Instrumentation Grant (S10OD016214). This work was supported by grants from the NIH (HL136662 to U.T.M., and R01 GM33397 to J.G.G.). J.G.G. is American Cancer Society Professor of Developmental Genetics. Research in the laboratory of D.L.J.L. is supported by the Belgian Fonds de la Recherche Scientifique (FRS/FNRS), the Université Libre de Bruxelles (ULB), the European Joint Programme on Rare Diseases ("RiboEurope" and "DBAGeneCure"), the Région Wallonne (SPW EER; "RIBOcancer"), the Internationale Brachet Stiftung, and the Epitran COST action (CA16120).

Author contributions: J.B. performed all experiments, except RNA modification analysis by primer extension, RT-PCR-based analysis, and RNase $\mathrm{H}$ cleavage, which were performed by S.D., and pre-rRNA processing experiments, which were performed by L.W. RNA-seq data were analyzed by V.G. and RiboMethSeq data were analyzed by F.G.M.E. U.T.M. conceived the study and wrote the manuscript, aided by J.B. The manuscript was edited by J.G.G., S.D., and D.L.J.L. J.G.G. and D.L.J.L. supervised the work in their laboratories. 


\section{References}

Bakin A, Ofengand J. 1993. Four newly located pseudouridylate residues in Escherichia coli 23S ribosomal RNA are all at the peptidyltransferase center: analysis by the application of a new sequencing technique. Biochemistry 32: 9754-9762. doi:10.1021/bi00088a030

Birkedal U, Christensen-Dalsgaard M, Krogh N, Sabarinathan R, Gorodkin J, Nielsen H. 2015. Profiling of ribose methylations in RNA by high-throughput sequencing. Angewandte Chemie Int Ed 54: 451-455.

Bizarro J, Bhardwaj A, Smith S, Meier UT. 2019. Nopp140-mediated concentration of telomerase in Cajal bodies regulates telomere length. Mol Biol Cell 30: 3136-3150. doi:10.1091/ mbc.E19-08-0429

Bouhaddou M, Memon D, Meyer B, White KM, Rezelj VV, Marrero MC, Polacco BJ, Melnyk JE, Ulferts S, Kaake RM, et al. 2020. The global phosphorylation landscape of SARS-CoV-2 infection. Cell 182: 685-712.e19. doi:10.1016/j.cell.2020.06 .034

Brangwynne CP, Mitchison TJ, Hyman AA. 2011. Active liquidlike behavior of nucleoli determines their size and shape in Xenopus laevis oocytes. Proc Natl Acad Sci 108: 4334-4339. doi:10.1073/pnas.1017150108

Chartrand P, Bertrand E, Singer RH, Long RM. 2000. [33] sensitive and high-resolution detection of RNA in situ. Methods Enzymol 318: 493-506. doi:10.1016/S0076-6879(00)18072-3

Chon HJ, Bae KJ, Lee Y, Kim J. 2015. The casein kinase 2 inhibitor, CX-4945, as an anti-cancer drug in treatment of human hematological malignancies. Front Pharmacol 6: 70.

Cozza G, Mazzorana M, Papinutto E, Bain J, Elliott M, di Maira G, Gianoncelli A, Pagano MA, Sarno S, Ruzzene M, et al. 2009. Quinalizarin as a potent, selective and cell-permeable inhibitor of protein kinase CK2. Biochem J 421: 387-395. doi:10 $.1042 / \mathrm{BJ} 20090069$

Darzacq X, Jády BE, Verheggen C, Kiss AM, Bertrand E, Kiss T. 2002. Cajal body-specific small nuclear RNAs: a novel class of 2'-O-methylation and pseudouridylation guide RNAs. EMBO J 21: 2746-2756. doi:10.1093/emboj/21.11.2746

Derenzini M, Thiry M, Goessens G. 1990. Ultrastructural cytochemistry of the mammalian cell nucleolus. I Histochem Cytochem 38: 1237-1256. doi:10.1177/38.9.2201735

Deryusheva S, Gall JG. 2009. Small Cajal body-specific RNAs of Drosophila function in the absence of Cajal bodies. Mol Biol Cell 20: 5250-5259. doi:10.1091/mbc.e09-09-0777

Deryusheva S, Gall JG. 2013. Novel small Cajal-body-specific RNAs identified in Drosophila: probing guide RNA function. RNA 19: 1802-1814. doi:10.1261/rna.042028.113

Deryusheva S, Gall JG. 2019. scaRNAs and snoRNAs: are they limited to specific classes of substrate RNAs? RNA 25: 1722. doi:10.1261/rna.068593.118

Deryusheva S, Choleza M, Barbarossa A, Gall JG, Bordonné R. 2012. Post-transcriptional modification of spliceosomal RNAs is normal in SMN-deficient cells. RNA 18: 31-36. doi:10.1261/rna.030106.111

Dobin A, Davis CA, Schlesinger F, Drenkow J, Zaleski C, Jha S, Batut P, Chaisson M, Gingeras TR. 2013. STAR: ultrafast universal RNA-seq aligner. Bioinformatics 29: 15-21. doi:10 .1093/bioinformatics/bts635

Dong Z-W, Shao P, Diao L-T, Zhou H, Yu C-H, Qu L-H. 2012. RTL-P: a sensitive approach for detecting sites of 2'-O-methylation in RNA molecules. Nucleic Acids Res 40: e157. doi:10 $.1093 / \mathrm{nar} / \mathrm{gks} 698$

Dönmez G, Hartmuth K, Lührmann R. 2004. Modified nucleotides at the $5^{\prime}$ end of human U2 snRNA are required for spli- ceosomal E-complex formation. RNA 10: 1925-1933. doi:10 $.1261 /$ rna. 7186504

Dundr M, Misteli T. 2001. Functional architecture in the cell nucleus. Biochem I 356: 297-310. doi:10.1042/bj3560297

Erales J, Marchand V, Panthu B, Gillot S, Belin S, Ghayad SE, Garcia M, Laforêts F, Marcel V, Baudin-Baillieu A, et al. 2017. Evidence for rRNA 2'-O-methylation plasticity: control of intrinsic translational capabilities of human ribosomes. Proc Natl Acad Sci 114: 12934-12939. doi:10.1073/pnas.170767 4114

Gall JG. 2003. The centennial of the Cajal body. Nat Rev Mol Cell Bio 4: 975-980. doi:10.1038/nrm1262

Ganot P, Jády BE, Bortolin M-L, Darzacq X, Kiss T. 1999. Nucleolar factors direct the 2'-O-ribose methylation and pseudouridylation of U6 spliceosomal RNA. Mol Cell Biol 19: 69066917. doi:10.1128/MCB.19.10.6906

Hadjiolov AA. 1985. The nucleolus and ribosome biogenesis. Cell Biology Monogr 12: 111-132. doi:10.1007/978-3-7091-87425_5

Hart T, Chandrashekhar M, Aregger M, Steinhart Z, Brown KR, MacLeod G, Mis M, Zimmermann M, Fradet-Turcotte A, Sun S, et al. 2015. High-Resolution CRISPR screens reveal fitness genes and genotype-specific cancer liabilities. Cell 163: 1515-1526. doi:10.1016/j.cell.2015.11.015

Hasler D, Meduri R, Bąk M, Lehmann G, Heizinger L, Wang X, Li Z-T, Sement FM, Bruckmann A, Dock-Bregeon A-C, et al. 2020. The alazami syndrome-associated protein LARP7 guides U6 small nuclear RNA modification and contributes to splicing robustness. Mol Cell 77: 1014-1031.e13. doi:10 .1016/j.molcel.2020.01.001

He F, James A, Raje H, Ghaffari H, DiMario P. 2015. Deletion of drosophila Nopp140 induces subcellular ribosomopathies. Chromosoma 124: 191-208. doi:10.1007/s00412-014-0490-9

Incarnato D, Anselmi F, Morandi E, Neri F, Maldotti M, Rapelli S, Parlato C, Basile G, Oliviero S. 2017. High-throughput singlebase resolution mapping of RNA 2'-O-methylated residues. Nucleic Acids Res 45: 1433-1441. doi:10.1093/nar/gkw810

Jády BE, Darzacq X, Tucker KE, Matera AG, Bertrand E, Kiss T. 2003. Modification of Sm small nuclear RNAs occurs in the nucleoplasmic Cajal body following import from the cytoplasm. EMBO I 22: 1878-1888. doi:10.1093/emboj/cdg187

Kiss T. 2001. New EMBO member's review: small nucleolar RNA-guided post-transcriptional modification of cellular RNAs. EMBO / 20: 3617-3622. doi:10.1093/emboj/20.14.3617

Kiss T. 2004. Biogenesis of small nuclear RNPs. J Cell Sci 117: 5949-5951. doi:10.1242/jcs.01487

Kittur N, Zapantis G, Aubuchon M, Santoro N, Bazett-Jones DP, Meier UT. 2007. The nucleolar channel system of human endometrium Is related to endoplasmic reticulum and R-rings. Mol Biol Cell 18: 2296-2304. doi:10.1091/mbc.e07-02-0154

Krogh N, Jansson MD, Häfner SJ, Tehler D, Birkedal U, Christensen-Dalsgaard M, Lund AH, Nielsen H. 2016. Profiling of 2'-OMe in human rRNA reveals a subset of fractionally modified positions and provides evidence for ribosome heterogeneity. Nucleic Acids Res 44: 7884-7895. doi:10.1093/nar/gkw482

Krogh N, Kongsbak-Wismann M, Geisler C, Nielsen H. 2017. Substoichiometric ribose methylations in spliceosomal snRNAs. Org Biomol Chem 15: 8872-8876. doi:10.1039/ C7OB02317K

Lafontaine DLJ, Riback JA, Bascetin R, Brangwynne CP. 2020. The nucleolus as a multiphase liquid condensate. Nat Rev Mol Cell Bio 22: 165-182. doi:10.1038/s41580-020-0272-6

Lapham J, Yu YT, Shu MD, Steitz JA, Crothers DM. 1997. The position of site-directed cleavage of RNA using RNase $\mathrm{H}$ and 2'- 
O-methyl oligonucleotides is dependent on the enzyme source. Rna New York N Y 3: 950-951.

Lestrade L, Weber MJ. 2006. snoRNA-LBME-db, a comprehensive database of human H/ACA and C/D box snoRNAs. Nucleic Acids Res 34: D158-D162. doi:10.1093/nar/gkj002

Liao Y, Smyth GK, Shi W. 2014. Featurecounts: an efficient general purpose program for assigning sequence reads to genomic features. Bioinformatics 30: 923-930. doi:10.1093/bioinfor matics/btt656

Love MI, Huber W, Anders S. 2014. Moderated estimation of fold change and dispersion for RNA-seq data with DESeq2. Genome Biol 15: 550. doi:10.1186/s13059-014-0550-8

Machyna M, Heyn P, Neugebauer KM. 2013. Cajal bodies: where form meets function. Wiley Interdiscip Rev Rna 4: 17-34. doi:10.1002/wrna.1139

Maden BEH, Corbett ME, Heeney PA, Pugh K, Ajuh PM. 1995. Classical and novel approaches to the detection and localization of the numerous modified nucleotides in eukaryotic ribosomal RNA. Biochimie 77: 22-29. doi:10.1016/0300-9084(96) 88100-4

Marchand V, Blanloeil-Oillo F, Helm M, Motorin Y. 2016. Illumina-based RiboMethSeq approach for mapping of 2'-O-Me residues in RNA. Nucleic Acids Res 44: e135. doi:10.1093/ nar/gkw547

Marchand V, Pichot F, Neybecker P, Ayadi L, Bourguignon-Igel V, Wacheul L, Lafontaine DLJ, Pinzano A, Helm M, Motorin Y. 2020. HydraPsiSeq: a method for systematic and quantitative mapping of pseudouridines in RNA. Nucleic Acids Res 48: e110. doi:10.1093/nar/gkaa769

Massenet S, Mougin A, Branlant C. 1998. Posttranscriptional modifications in the U small nuclear RNAs. In Modification and editing of RNA (ed. Grosjean H, Benne R), pp. 201-227. American Society of Microbiology, Washington, DC. doi:10 $.1128 / 9781555818296 . c h 11$

Massenet S, Bertrand E, Verheggen C. 2017. Assembly and trafficking of box C/D and H/ACA snoRNPs. Rna Biol 14: 680692. doi:10.1080/15476286.2016.1243646

Maxwell ES, Fournier MJ. 1995. The small nucleolar RNAs. Annu Rev Biochem 64: 897-934. doi:10.1146/annurev.bi.64.070195 .004341

McPheeters DS, Fabrizio P, Abelson J. 1989. In vitro reconstitution of functional yeast U2 snRNPs. Gene Dev 3: 21242136. doi:10.1101/gad.3.12b.2124

Meier UT. 1996. Comparison of the rat nucleolar protein Nopp140 with its yeast homolog SRP40: differential phosphorylation in vertebrates and yeast. Journal of Biological Chemistry 271: 19376-19384. doi:10.1016/S0021-9258(19)84375-2

Meier UT. 2005. The many facets of H/ACA ribonucleoproteins. Chromosoma 114: 1-14. doi:10.1007/s00412-005-0333-9

Meier UT. 2017. RNA modification in Cajal bodies. Rna Biol 14: 693-700. doi:10.1080/15476286.2016.1249091

Meier UT, Blobel G. 1990. A nuclear localization signal binding protein in the nucleolus. I Cell Biology 111: 2235-2245. doi: $10.1083 /$ jcb.111.6.2235

Meier UT, Blobel G. 1992. Nopp140 shuttles on tracks between nucleolus and cytoplasm. Cell 70: 127-138. doi:10.1016/ 0092-8674(92) 90539-O

Meier UT, Blobel G. 1994. NAP57, a mammalian nucleolar protein with a putative homolog in yeast and bacteria. I Cell Biol 127: 1505-1514. doi:10.1083/jcb.127.6.1505

Morais P, Adachi H, Yu Y-T. 2021. Spliceosomal snRNA epitranscriptomics. Frontiers Genetics 12: 652129. doi:10.3389/fgene .2021 .652129

Na J-H, Lee W-K, Yu YG. 2018. How do we study the dynamic structure of unstructured proteins: a case study on Nopp140 as an example of a large, intrinsically disordered protein. Int J Mol Sci 19: 381. doi:10.3390/ijms19020381

Neugebauer KM. 2017. Special focus on the Cajal body. Rna Biol 14: 669-670. doi:10.1080/15476286.2017.1316928

Scheer U, Hock R. 1999. Structure and function of the nucleolus. Curr Opin Cell Biol 11: 385-390. doi:10.1016/S0955-0674(99) 80054-4

Scheer U, Hügle B, Hazan R, Rose KM. 1984. Drug-induced dispersal of transcribed rRNA genes and transcriptional products: immunolocalization and silver staining of different nucleolar components in rat cells treated with 5,6-dichloro$\beta$-D-ribofuranosylbenzimidazole. I Cell Biol 99: 672-679. doi:10.1083/jcb.99.2.672

Sharma S, Langhendries J-L, Watzinger P, Kötter P, Entian K-D, Lafontaine DLJ. 2015. Yeast Kre33 and human NAT10 are conserved 18S rRNA cytosine acetyltransferases that modify tRNAs assisted by the adaptor Tan1/THUMPD1. Nucleic Acids Res 43: 2242-2258. doi:10.1093/nar/gkv075

Sharma S, Marchand V, Motorin Y, Lafontaine DLJ. 2017. Identification of sites of 2'-O-methylation vulnerability in human ribosomal RNAs by systematic mapping. Sci Rep 7: 11490 . doi:10.1038/s41598-017-09734-9

Shen S, Park JW, Lu Z, Lin L, Henry MD, Wu YN, Zhou Q, Xing Y. 2014. rMATS: robust and flexible detection of differential alternative splicing from replicate RNA-seq data. Proc Natl Acad Sci 111: E5593-E5601. doi:10.1073/pnas.1419161111

Siddiqui-Jain A, Drygin D, Streiner N, Chua P, Pierre F, O'Brien SE, Bliesath J, Omori M, Huser N, Ho C, et al. 2010. CX4945 , an orally bioavailable selective inhibitor of protein kinase CK2, inhibits prosurvival and angiogenic signaling and exhibits antitumor efficacy. Cancer Res 70: 10288-10298. doi:10.1158/0008-5472.CAN-10-1893

Silva-Pavez E, Tapia JC. 2020. Protein kinase CK2 in cancer energetics. Frontiers Oncol 10: 893. doi:10.3389/fonc.2020.00893

Smith CM, Steitz JA. 1997. Sno storm in the nucleolus: new roles for myriad small RNPs. Cell 89: 669-672. doi:10.1016/S00928674(00) 80247-0

Spector DL. 1993. Macromolecular domains within the cell nucleus. Annu Rev Cell Biol 9: 265-315. doi:10.1146/annurev .cb.09.110193.001405

Spector DL, Lark G, Huang S. 1992. Differences in snRNP localization between transformed and nontransformed cells. Mol Biol Cell 3: 555-569. doi:10.1091/mbc.3.5.555

Tafforeau L, Zorbas C, Langhendries J-L, Mullineux S-T, Stamatopoulou V, Mullier R, Wacheul L, Lafontaine DLJ. 2013. The complexity of human ribosome biogenesis revealed by systematic nucleolar screening of pre-rRNA processing factors. Mol Cell 51: 539-551. doi:10.1016/j.molcel.2013.08.011

Tantos A, Szrnka K, Szabo B, Bokor M, Kamasa P, Matus P, Bekesi A, Tompa K, Han K-H, Tompa P. 2013. Structural disorder and local order of hNopp140. Biochimica Et Biophysica Acta BbaProteins Proteom 1834: 342-350. doi:10.1016/j.bbapap.2012 .08 .005

Taoka M, Nobe Y, Yamaki Y, Sato K, Ishikawa H, Izumikawa K, Yamauchi Y, Hirota K, Nakayama H, Takahashi N, et al. 2018. Landscape of the complete RNA chemical modifications in the human 80S ribosome. Nucleic Acids Res 46: 9289-9298. doi:10.1093/nar/gky811

Townsend C, Leelaram MN, Agafonov DE, Dybkov O, Will CL, Bertram K, Urlaub H, Kastner B, Stark H, Lührmann R. 2020. Mechanism of protein-guided folding of the active site U2/U6 RNA during spliceosome activation. Science 370: eabc3753. doi:10.1126/science.abc3753

Trapnell C, Williams BA, Pertea G, Mortazavi A, Kwan G, Baren MJ van, Salzberg SL, Wold BJ, Pachter L. 2010. Transcript 
assembly and quantification by RNA-seq reveals unannotated transcripts and isoform switching during cell differentiation. Nat Biotechnol 28: 511-515. doi:10.1038/nbt.1621

Tucker KE, Berciano MT, Jacobs EY, LePage DF, Shpargel KB, Rossire JJ, Chan EKL, Lafarga M, Conlon RA, Matera AG. 2001. Residual Cajal bodies in coilin knockout mice fail to recruit $\mathrm{Sm}$ snRNPs and SMN, the spinal muscular atrophy gene product. J Cell Biology 154: 293-308. doi:10.1083/jcb.200104083

Waggener JM, DiMario PJ. 2002. Two splice variants of Nopp140 in Drosophila melanogaster. Mol Biol Cell 13:362-381. doi:10 .1091/mbc.01-04-0162

Wang C, Query CC, Meier UT. 2002. Immunopurified small nucleolar ribonucleoprotein particles pseudouridylate rRNA independently of their association with phosphorylated Nopp140. Mol Cell Biol 22: 8457-8466. doi:10.1128/MCB.22 .24.8457-8466.2002

Wang T, Birsoy K, Hughes NW, Krupczak KM, Post Y, Wei JJ, Lander ES, Sabatini DM. 2015. Identification and characterization of essential genes in the human genome. Science 350: 1096-1101. doi:10.1126/science.aac7041

Wilkinson ME, Charenton C, Nagai K. 2019. RNA splicing by the spliceosome. Annu Rev Biochem 89: 359-388. doi:10.1146/ annurev-biochem-091719-064225

Wu G, Adachi H, Ge J, Stephenson D, Query CC, Yu Y. 2016. Pseudouridines in U2 snRNA stimulate the ATPase activity of Prp5 during spliceosome assembly. Embo I 35: 654-667. doi:10.15252/embj.201593113
Yu Y-T, Shu MD, Steitz JA. 1997. A new method for detecting sites of 2'-O-methylation in RNA molecules. RNA 3: 324-331.

Yu Y, Shu M, Steitz JA. 1998. Modifications of U2 snRNA are required for snRNP assembly and pre-mRNA splicing. Embo J 17: 5783-5795. doi:10.1093/emboj/17.19.5783

Yu G, Wang L-G, Han Y, He Q-Y. 2012. Clusterprofiler: an R package for comparing biological themes among gene clusters. Omics I Integr Biology 16: 284-287. doi:10.1089/omi.2011 .0118

Zhang X, Yan C, Hang J, Finci LI, Lei J, Shi Y. 2017. An atomic structure of the human spliceosome. Cell 169: 918-929.e14. doi:10.1016/j.cell.2017.04.033

Zhang Z, Will CL, Bertram K, Dybkov O, Hartmuth K, Agafonov DE, Hofele R, Urlaub H, Kastner B, Lührmann R, et al. 2020. Molecular architecture of the human 17S U2 snRNP. Nature 583: 310-313. doi:10.1038/s41586-020-2344-3

Zhao X, Yu Y-T. 2004. Pseudouridines in and near the branch site recognition region of U2 snRNA are required for snRNP biogenesis and pre-mRNA splicing in xenopus oocytes. RNA 10: 681-690. doi:10.1261/rna.5159504

Zhao X, Yu Y-T. 2007. Incorporation of 5-fluorouracil into U2 snRNA blocks pseudouridylation and pre-mRNA splicing in vivo. Nucleic Acids Res 35: 550-558. doi:10.1093/nar/gk11084

Zhu L, Brangwynne CP. 2015. Nuclear bodies: the emerging biophysics of nucleoplasmic phases. Curr Opin Cell Biol 34: 23-30. 


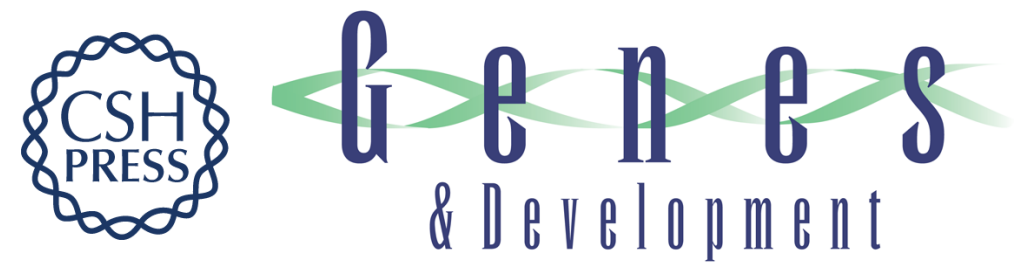

\section{Nopp140-chaperoned 2'-O-methylation of small nuclear RNAs in Cajal bodies ensures splicing fidelity}

Jonathan Bizarro, Svetlana Deryusheva, Ludivine Wacheul, et al.

Genes Dev. published online July 22, 2021

Access the most recent version at doi:10.1101/gad.348660.121

\section{Supplemental http://genesdev.cshlp.org/content/suppl/2021/07/21/gad.348660.121.DC1 Material}

Published online July 22, 2021 in advance of the full issue.

Creative This article is distributed exclusively by Cold Spring Harbor Laboratory Press for the first Commons License six months after the full-issue publication date (see

http://genesdev.cshlp.org/site/misc/terms.xhtml). After six months, it is available under a Creative Commons License (Attribution-NonCommercial 4.0 International), as described at http://creativecommons.org/licenses/by-nc/4.0/.

Email Alerting

Receive free email alerts when new articles cite this article - sign up in the box at the top Service right corner of the article or click here. 